\title{
aM-Conotoxin MIIIJ Blocks Nicotinic Acetylcholine Receptors at Neuromuscular Junctions of Frog and Fish
}

\author{
Matthew J. Rybin ${ }^{1,2} \oplus$, Henrik $\mathrm{O}^{\prime}$ Brien $^{1}{ }^{1}$, Iris Bea L. Ramiro ${ }^{1,3}{ }^{\oplus}$, Layla Azam ${ }^{1}$, \\ J. Michael McIntosh 1,4,5, Baldomero M. Olivera ${ }^{1}$, Helena Safavi-Hemami 1,3,6,*(D) and \\ Doju Yoshikami ${ }^{1, *}$ \\ 1 Department of Biology, University of Utah, Salt Lake City, UT 84112, USA; mxr2011@miami.edu (M.J.R.); \\ henrikobrien@gmail.com (H.O.); iris.ramiro@sund.ku.dk (I.B.L.R.); layla.azam@utah.edu (L.A.); \\ michael.mcintosh@utah.edu (J.M.M.); olivera@biology.utah.edu (B.M.O.) \\ 2 Department of Psychiatry and Behavioral Sciences, University of Miami, Miller School of Medicine, Miami, \\ FL 33136, USA \\ 3 Department of Biomedical Sciences, University of Copenhagen, 2200 Copenhagen, Denmark \\ 4 George E. Whalen Veterans Affairs Medical Center, Salt Lake City, UT 84112, USA \\ 5 Department of Psychiatry, University of Utah, Salt Lake City, UT 84112, USA \\ 6 Department of Biochemistry, University of Utah, Salt Lake City, UT 84112, USA \\ * Correspondence: safavihelena@sund.ku.dk (H.S.-H.); yoshikami@bioscience.utah.edu (D.Y.)
}

Received: 22 February 2020; Accepted: 18 March 2020; Published: 21 March 2020

check for updates

\begin{abstract}
We report the discovery and functional characterization of $\alpha \mathrm{M}$-Conotoxin MIIIJ, a peptide from the venom of the fish-hunting cone snail Conus magus. Injections of $\alpha \mathrm{M}-\mathrm{MIIIJ}$ induced paralysis in goldfish (Carassius auratus) but not mice. Intracellular recording from skeletal muscles of fish (C. auratus) and frog (Xenopus laevis) revealed that $\alpha \mathrm{M}-\mathrm{MIIIJ}$ inhibited postsynaptic nicotinic acetylcholine receptors (nAChRs) with an $\mathrm{IC}_{50}$ of $\sim 0.1 \mu \mathrm{M}$. With comparable potency, $\alpha \mathrm{M}-\mathrm{MIIIJ}$ reversibly blocked ACh-gated currents $\left(\mathrm{I}_{\mathrm{ACh}}\right)$ of voltage-clamped X. laevis oocytes exogenously expressing $\mathrm{nAChRs}$ cloned from zebrafish (Danio rerio) muscle. $\alpha \mathrm{M}-\mathrm{MIIIJ}$ also protected against slowly-reversible block of $\mathrm{I}_{\mathrm{ACh}}$ by $\alpha$-bungarotoxin ( $\alpha$-BgTX, a snake neurotoxin) and $\alpha$-conotoxin EI ( $\alpha$-EI, from Conus ermineus another fish hunter) that competitively block nAChRs at the ACh binding site. Furthermore, assessment by fluorescence microscopy showed that $\alpha \mathrm{M}-\mathrm{MIIIJ}$ inhibited the binding of fluorescently-tagged $\alpha$-BgTX at neuromuscular junctions of X. laevis, C. auratus, and D. rerio. (Note, we observed that $\alpha \mathrm{M}-\mathrm{MIIIJ}$ can block adult mouse and human muscle $n A C h R s$ exogenously expressed in X. laevis oocytes, but with $\mathrm{IC}_{50} \mathrm{~s} \sim 100$-times higher than those of zebrafish nAChRs.) Taken together, these results indicate that $\alpha \mathrm{M}-\mathrm{MIIIJ}$ inhibits muscle $\mathrm{nAChRs}$ and furthermore apparently does so by interfering with the binding of ACh to its receptor. Comparative alignments with homologous sequences identified in other fish hunters revealed that $\alpha \mathrm{M}-\mathrm{MIIIJ}$ defines a new class of muscle nAChR inhibitors from cone snails.
\end{abstract}

Keywords: venom; conotoxin; nicotinic acetylcholine receptor; neuromuscular junction

Key Contribution: We report the discovery and neurophysiological characterization of the first member of a class of muscle nAChR antagonists from venomous marine cone snails.

\section{Introduction}

Cone snail venoms have a profusion of peptides that target ion channels of the nervous system [1-3]. Owing to their diversity and target specificity, cone snail toxins (conotoxins) have become valuable 
tools for ion channel research and drug design [3-5]. Cone snails form a diverse genus of $\sim 800$ extant species that have evolved diverse diets and predation strategies. Conus magus belongs to one of the most studied clades of fish-hunting cone snails, the Pionoconus clade. Pionoconus cone snails use the taser-and-tether hunting strategy that is characterized by rapid, irreversible paralysis following venom injection [6]. This is achieved by the concerted action of toxins that target ion channels and receptors of the peripheral nervous system, particularly those at the neuromuscular junction [7]. Many of the paralytic components of Pionoconus venom have been characterized. For example, the venom of the fish hunter Conus magus has three families of paralytic peptides, and a representative member of each family is as follows: $\alpha$-conotoxin MI, which blocks postsynaptic nicotinic acetylcholine receptors (nAChRs) that are essential for excitatory postsynaptic potentials (EPSPs) in muscle fibers; $\omega$-conotoxin MVIIA, which blocks voltage-gated calcium channels (V-gated Ca channels) whose activity triggers transmitter (acetylcholine, $\mathrm{ACh}$ ) release from the presynaptic motor-nerve terminal; and $\mu$-conotoxin MIIIA, which blocks voltage-gated sodium channels (V-gated Na channels) responsible for action potentials in muscle fibers (see Table 1 for peptide sequences and references). Here, we describe the identification of $\alpha \mathrm{M}-\mathrm{MIIIJ}$, the first member of a previously uncharacterized family of paralytic toxins with functional but no structural similarity to $\alpha$-conotoxin MI.

Table 1. Sequences and targets of paralytic conotoxins from C. magus.

\begin{tabular}{llll}
\hline Peptide & Sequence $^{1}$ & Ion-Channel Target & Reference \\
\hline$\alpha$-MI & GRCCHPACGKNYSC\# & muscle nAChR & McIntosh 1982 [8] \\
$\alpha$-MII ${ }^{2}$ & GCCSNPVCHLEHSNLC\# & neuronal nAChR & Cartier 1996 [9] \\
$\omega$-MVIIA & CKGKGAKCSRLMYDCCTGSCRSGKC\# & presynaptic Ca channel & Olivera 1987 [10] \\
$\mu$-MIIIA & ZGCCNVPNGCSGRWCRDHAQCC\# & muscle Na channel & Wilson 2011 [11] \\
$\alpha$ M-MIII & ZKCCSGGSCPLYFRDRLICPCC & muscle nAChR & Zhang 2006 [12] \\
\hline
\end{tabular}

${ }^{1}$ Cysteine residues are bolded to highlight them; \# indicates C-terminal amidation; $\mathrm{Z}$ indicates pyroglutamate residue. ${ }^{2} \alpha$-MII is not paralytic but is shown here for comparison with $\alpha$-MI.

$\alpha \mathrm{M}-\mathrm{MIIIJ}$ was originally identified by fractionation of $C$. magus venom and sequencing of some of its major components during the purification and characterization of $\alpha$-MI by McIntosh et al. in 1982 [8]. Because $\alpha \mathrm{M}-\mathrm{MIIIJ}$ did not elicit a visible phenotype upon intracranial injections into mice and was inactive in blocking synaptic transmission at the neuromuscular junction of the grass frog, Rana pipiens, the pharmacological characterization of this toxin was not further pursued, and the discovery of this peptide not reported.

Here, using electrophysiological experiments, we demonstrate that $\alpha \mathrm{M}-\mathrm{MIIIJ}$ blocks the function of nAChRs at the neuromuscular junctions (NMJs) of African clawed frog (Xenopus laevis) and goldfish (Carassius auratus). We confirm that $\alpha$ M-MIIIJ does not block $n A C h R s$ at the NMJ of R. pipiens. We also show that $\alpha \mathrm{M}-\mathrm{MIIIJ}$ reversibly blocks muscle $\mathrm{nAChRs}$ cloned from zebrafish (Danio rerio) and exogenously expressed in X. laevis oocytes; moreover, $\alpha \mathrm{M}$-MIIIJ protects these receptors from block by $\alpha$-bungarotoxin $(\alpha$-BgTX) and $\alpha$-conotoxin EI $(\alpha$-EI). We believe this is the first report where an nAChR-targeting conotoxin from a fish hunter has been functionally tested on molecularly-defined fish nAChRs. Finally, we show by fluorescence microscopy that $\alpha \mathrm{M}-\mathrm{MIIIJ}$ inhibits the binding of fluorescently-labeled $\alpha$-BgTX to nAChRs at NMJs of frog (X. laevis, but not R. pipiens) and both goldfish and zebrafish. Mining of published transcriptome data of C. magus and related cone snail species suggests that $\alpha \mathrm{M}-\mathrm{MIIIJ}$ is the first functionally characterized member of a large class of toxins expressed in taser-and-tether cone snails.

\section{Results}

\section{1. $\alpha M-M I I I J$ Discovery}

$\alpha \mathrm{M}-\mathrm{MIIIJ}$ was discovered, albeit not reported, during our initial purification of $\alpha-\mathrm{MI}$ [8]. Upon reversed-phase fractionation of crude venom from C. magus, $\alpha$ M-MIIIJ eluted considerably later 
than $\alpha$-MI (Figure S1). Further purification and sequencing of the reversed-phase fraction led to the structural characterization of $\alpha \mathrm{M}$-MIIIJ. $\alpha \mathrm{M}$-MIIIJ has 22 amino acids including an N-terminal pyro-glutamate (Figure 1A), and a molecular mass of 2429 Da (see below). Furthermore, $\alpha \mathrm{M}-\mathrm{MIIIJ}$ has six cysteines connected by three disulfide bonds and exhibits a type III cysteine framework (CC-C-C-CC) [13]. Mining of the venom gland transcriptome of C. magus and two other species from the Pionoconus clade, Conus consors and Conus striatus, identified several toxins with high sequence similarity to $\alpha \mathrm{M}-\mathrm{MIIIJ}$ (Figure 1A). Of these, the C. consors peptides (CnIIIE, CnIIIF, CnIIIG) were previously shown to be part of the injected venom cocktail of this species, but their activity was not explored [14].

A

aIIIJ
MIIIK
MIIIL
CnIIIE
CnIIIF
CnIIIG
SIIIJ

$\mu \mathrm{BuIIIB}$

$\mu \mathrm{KIIIA}$

$\mu \mathrm{MIIIA}$

$\mu \mathrm{GIIIA}$

UPIIIE

UPrIIIE
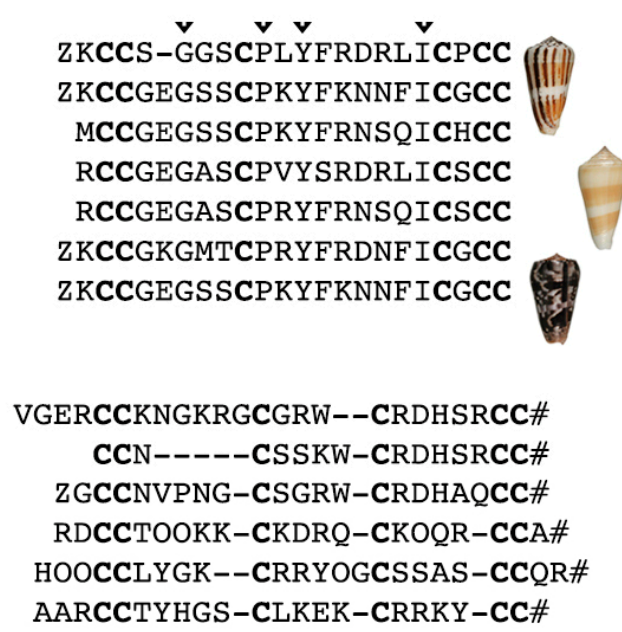

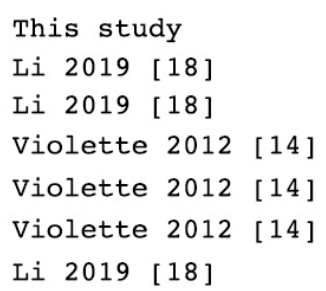

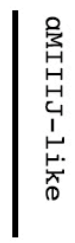

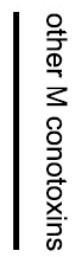

\section{B}

aMIIIJ MIIIK

CnIIIE

BuIIIB RIIIK
Signal sequence

Propeptide
Toxin

ZKCC-SGGS-CPLYFRDRLICPCC MSKLGVLLTICLLLFPLTALPMDEDQPADQLEDRMQDDISSEQYPSFVRR--QKCCGEGSS-CPKYFKNNFICGCC MSKLGVLLTICLLLFPLTALPMDGDOSVDRPAERMODDISSEOYPLFNOK--RRCCGEGAS-CPVYSRDRLICSCC MSKLGVLLTICLLLFPLFALPQDGDQPADRPAERMQDDISSEQNPLLEKRVGERCCKNGKRGCGRWCRDHSRCCGRR MSKLGVLLTICLLLFPLTALPMDGDQPVDRLAERMQDNISSEQHTFFEKRL-PSCCSLNLRLCPVPACKRNPCCTG $* * * * * * * * * * * * * * * * * * * * * . . *: \quad: * * * * ; * * * * .::: \quad * *: \quad *$

Figure 1. Sequence of $\alpha \mathrm{M}-\mathrm{MIIIJ}$ compared with those of other conotoxins. A. Sequence alignment of $\alpha \mathrm{M}-\mathrm{MIIIJ}$ with related sequences of unknown activity identified in C. magus, C. consors, and C. striatus (top) and M-superfamily conotoxins (bottom) that block V-gated Na channels ( $\mu$-prefix) or nAChRs ( $\psi$-prefix for binding at a noncompetitive site). Cysteines are in bold. Identical amino acids are shown with arrowhead on top of $\alpha$ M-MIIIJ sequence. Z: pyroglutamic acid, O: hydroxyproline, \#: C-terminal amidation. References [12,14-20]. B. Precursor sequence alignment of M-superfamily toxins highlights a conserved signal sequence used for toxin gene classification. The precursor sequence of $\alpha \mathrm{M}-\mathrm{MIIIJ}$ could not be retrieved, but high sequence similarities with $\alpha \mathrm{M}$-MIIIJ-like sequences, including MIIIK (91\%), strongly suggest that $\alpha \mathrm{M}-\mathrm{MIIIJ}$ also belongs to the M-superfamily. Amino acid conservations are denoted by an asterisk $\left({ }^{*}\right)$. Full stops (.) and colons (:) represent a low and high degree of similarity, respectively.

According to their conserved N-terminal signal sequence, conotoxins can be grouped into genetically related toxin superfamilies [3]. While we were not able to retrieve the precursor sequence of $\alpha \mathrm{M}-\mathrm{MIIIJ}$, the full-length precursors of highly similar sequences could be unambiguously assigned to the M-superfamily (Figure 1B) [21] strongly suggesting that $\alpha \mathrm{M}$-MIIIJ is also a member of the M-superfamily. For example, $\alpha$ M-MIIIJ and MIIIK share 74\% sequence identity and $91 \%$ sequence similarity. The absence of an identical sequence to $\alpha \mathrm{M}-\mathrm{MIIIJ}$ in the published transcriptome data of C. magus can be attributed to the high intraspecies sequence variation of cone snail toxins. The venom used in our study was not extracted from the same specimen as that used for transcriptome sequencing. 
Thus, we did not necessarily anticipate finding an exact sequence match but believe that the difference in amino acid sequence is due to intraspecies variation. $\alpha \mathrm{M}-\mathrm{MIIIJ}$ was named according to the conventional conotoxin nomenclature, where the Greek letter represents the pharmacological class (" $\alpha$ " for inhibitors of nAChRs), followed by a single letter for the gene family (" $\mathrm{M}$ " for $\underline{\mathrm{M}}$-superfamily), one or two letters representing the species name (" $\mathrm{M}$ " for magus), a Roman numeral for the cysteine framework ("III") and a letter or number given to identify different toxins belonging to the same class ("J").

\section{2. $\alpha M-M I I I J$ Synthesis}

Solid-phase synthesis was carried out as described in Methods. The final product showed $98 \%$ purity by analytical HPLC. The observed monoisotopic $(\mathrm{M}+\mathrm{H})^{+}$value of the synthetic peptide was 2428.6, which corresponded with the calculated $(\mathrm{M}+\mathrm{H})^{+}$value of 2429.0. The peptide was co-injected with the venom-purified peptide and analyzed by capillary zone electrophoresis (CZE); there was no difference in the migration between the native and the synthetic peptide.

\subsection{Paralytic Effects of $\alpha M-M I I I J$}

In-vivo assays revealed that $\alpha \mathrm{M}$-MIIIJ paralyzed goldfish (Table S1) but not mice. Three Swiss Webster mice $(22.34,8.65,8.52 \mathrm{~g})$ were injected intraperitoneally (i.p.) with $\alpha \mathrm{M}-\mathrm{MIIIJ}(\sim 2.3 \mathrm{nmol} / \mathrm{g}$ mouse) and observed without any reduced motor function or paralysis for $>1.5 \mathrm{~h}$. For comparison, a mouse ( $8.28 \mathrm{~g}$ ) was injected i.p. with $\alpha$-conotoxin EI ( $\alpha$-EI, $0.24 \mathrm{nmol} / \mathrm{g}$ mouse), an antagonist of muscle nAChRs [22], that caused paralysis and then death in $<10 \mathrm{~min}$. This initial observation suggested muscle nAChR may not be the molecular target of $\alpha \mathrm{M}-\mathrm{MIIIJ}$, at least not in mice. To identify its molecular target, we examined the effect of $\alpha \mathrm{M}-\mathrm{MIIIJ}$ on isolated muscle preparations from lower vertebrates, namely two species each of frog and fish as well as on cloned muscle nAChRs of zebrafish exogenously expressed in X. laevis oocytes.

\subsection{Extracellular Electrophysiology of Frog Muscle Preparations}

$\alpha \mathrm{M}-\mathrm{MIIIJ}$ readily blocked extracellularly-recorded action potentials in X. laevis longitudinal pectoralis (LP) muscle evoked by motor-nerve stimulation (Figure 2A) but not those evoked by direct muscle stimulation (Figure 2B). The resistance of directly-evoked action potentials to $\alpha \mathrm{M}$-MIIIJ indicates that V-gated $\mathrm{Na}$ channels in the muscle were not blocked by the peptide. To examine the susceptibility of synaptic potentials to the peptide, preparations were treated with $\mu$-PIIIA, a conotoxin that irreversibly blocks V-gated Na channels in frog muscle [23]; thus, only synaptic potentials are observed upon nerve stimulation. Such synaptic potentials were readily blocked by $10 \mu \mathrm{M} \alpha \mathrm{M}-\mathrm{MIIIJ}$ (Figure 2C). This result suggests that $\alpha \mathrm{M}$-MIIIJ blocked a target that directly mediated synaptic transmission; for example, either a presynaptic voltage-gated calcium channel or postsynaptic nAChR. 

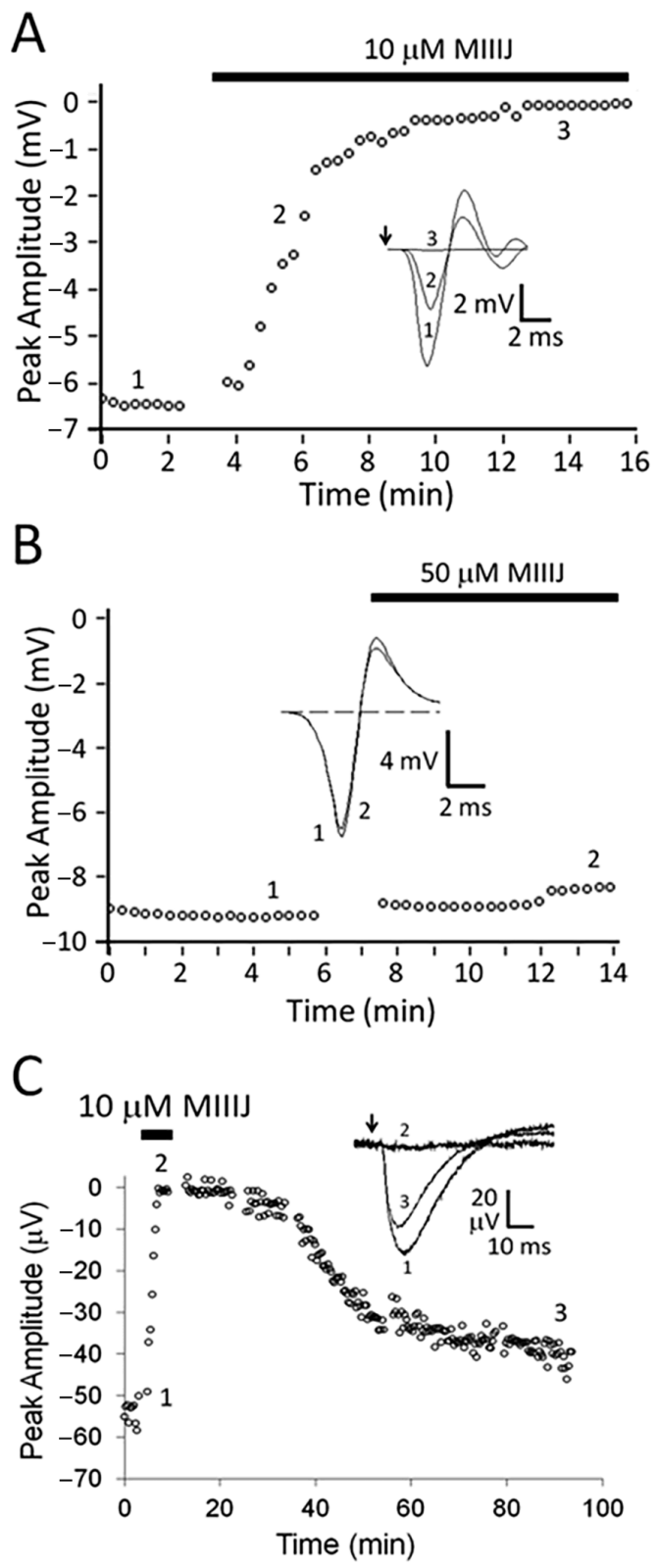

Figure 2. $\alpha \mathrm{M}-\mathrm{MIIIJ}$ blocks excitatory synaptic responses but not action potentials in X. laevis longitudinal pectoralis (LP) muscle. Extracellular recording from adult muscle was performed as described in Methods. Graphs plot peak amplitudes of responses versus time, where presence of $\alpha \mathrm{M}-\mathrm{MIIIJ}$ is represented by black bar above each plot and insets show representative traces at the enumerated time points. Arrows in insets of panels $A$ and $C$ show when stimulus was applied. A and B. Muscle action potentials in response to indirect (A) and direct (B) stimulation. Ten $\mu \mathrm{M} \alpha \mathrm{M}-\mathrm{MIIIJ}$ completely blocked action potentials evoked by nerve stimulation (A); in contrast, a five-times higher concentration of $\alpha \mathrm{M}-\mathrm{MIIIJ}$ had essentially no effect on action potentials evoked by direct stimulation (B). As a positive control, $\mu$-PIIIA $(10 \mu \mathrm{M})$, a conotoxin that irreversibly inhibits muscle V-gated Na channels, completely blocked action potentials within $10 \mathrm{~min}$. (flat dashed trace of panel B's inset). C. Motor-nerve stimulation evoked synaptic responses (without confounding muscle action potentials) in muscle preparation treated with $\mu$-PIIIA $(10 \mu \mathrm{M})$. The synaptic responses were reversibly blocked by $10 \mu \mathrm{M}$ $\alpha \mathrm{M}-\mathrm{MIIIJ}$. Each trace in inset of panel C represents the average of $\sim 10$ responses. Results replicating those illustrated here (i.e., complete block by toxin of nerve stimulation-evoked action potentials and synaptic responses, and conversely no block by toxin of direct stimulation-evoked action potentials) were obtained in three other LP muscle preparations. 
When R. pipiens muscles were tested, $\alpha \mathrm{M}-\mathrm{MIIIJ}$ did not block responses evoked by indirect stimulation (Figure 3). The persistence of the muscle action potential (Figure 3A) meant that V-gated $\mathrm{Na}$ channels were not blocked; moreover, this result also implied that the underlying synaptic response that triggered the action potential was also insensitive to $\alpha \mathrm{M}-\mathrm{MIIIJ}$. The latter implication was examined more closely by looking directly at synaptic responses in muscles pre-treated with $\mu$-PIIIA. Indeed, synaptic potentials in R. pipiens muscle were resistant to block by $\alpha \mathrm{M}-\mathrm{MIIIJ}$ even at a 10-fold higher concentration than that which rapidly obliterated synaptic potentials in X. laevis muscle (compare Figure 3B with Figure 2C).

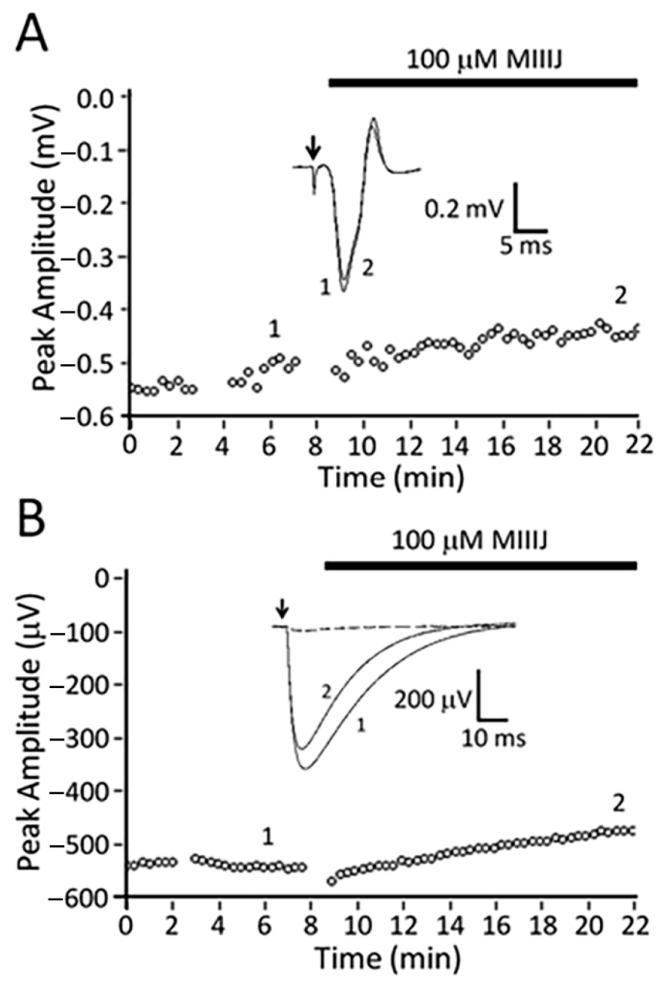

Figure 3. Action and synaptic potentials in R. pipiens cutaneous pectoralis $(\mathrm{CP})$ muscle are unaffected by $\alpha \mathrm{M}-\mathrm{MIIIJ}(100 \mu \mathrm{M})$. Muscle preparation and recording were as described in Methods. Format of presentation of results are as in Figure 2 (except traces in panel B were acquired with a low pass-filter setting of 0.1 , instead of $1, \mathrm{~Hz}$ ). Indirectly-evoked action potentials (A) and synaptic responses (B) are essentially unaffected by $100 \mu \mathrm{M} \alpha \mathrm{M}$-MIIIJ. In B, the muscle was treated with $\mu$-PIIIA as in Figure $2 \mathrm{C}$. As a positive control, the preparation was exposed to the muscle nAChR antagonist d-tubocurare $(10 \mu \mathrm{M})$, which blocked within $15 \mathrm{~min}$. (largely flat, dashed trace of panel B's inset). These results indicate that $\alpha \mathrm{M}-\mathrm{MIIIJ}$ does not block the muscle action potential in R. pipiens (like in X. laevis) muscle, nor does $\alpha \mathrm{M}$-MIIIJ block (unlike in X. laevis) the synaptically-evoked response. Results replicating those illustrated here (i.e., no block of either action or synaptic potentials by toxin) were obtained in three other CP muscle preparations.

To examine whether synaptic block by $\alpha \mathrm{M}$-MIIIJ in the X. laevis preparation was pre- or post-synaptic, intracellular recordings were made from muscles pretreated with $\mu$-PIIIA to abolish muscle twitches normally evoked by motor nerve stimulation. In addition, preparations were treated with fluorescein-peanut agglutinin (f-PNA) to locate NMJs to assist in the placement of the intracellular recording electrode and the ACh-iontophoresis electrode, as described in Methods. $\alpha \mathrm{M}-\mathrm{MIIIJ}$ blocked nerve-stimulation evoked EPSPs (Figure 4A), spontaneous miniature EPSPs (MEPSPs, Figure 4B) and responses to iontophoreticaly-applied ACh (Figure 4C). $\alpha \mathrm{M}$-MIIIJ blocked these responses $>50 \%$ at a concentration of $0.2 \mu \mathrm{M}$, and $100 \%$ at a concentration of $10 \mu \mathrm{M}$. Block of EPSPs, MEPSPs 
and ACh-evoked reponses was also observed at other $\alpha \mathrm{M}-\mathrm{MIIIJ}$ concentrations on separate muscle preparations (see Figure 6). These results clearly show that $\alpha \mathrm{M}-\mathrm{MIIIJ}$ blocks $\mathrm{nAChRs}$ in X. laevis muscle.
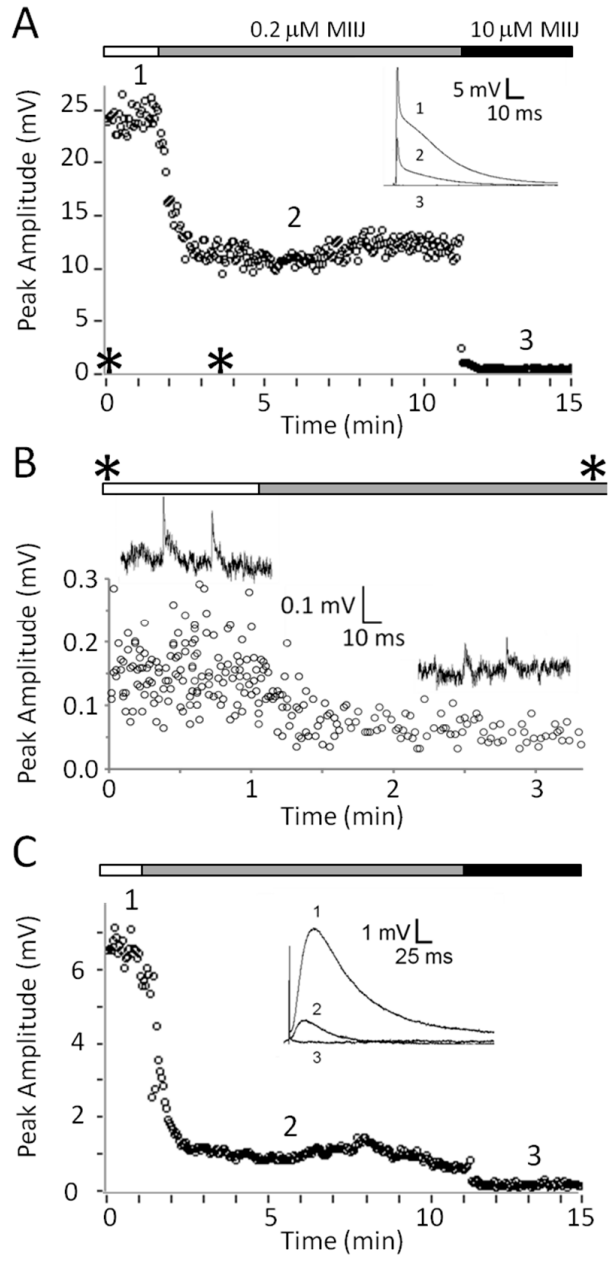

Figure 4. $\alpha \mathrm{M}$-MIIIJ blocks excitatory postsynaptic potentials (EPSPs), miniature excitatory postsynaptic potentials (MEPSPs), and ACh-evoked postsynaptic potentials (PSPs) in X. laevis LP muscle. Intracellular recording from juvenile muscle preparation was performed as described in Methods. All responses were obtained contemporaneously from one muscle fiber whose resting potential ranged between -77 to $-88 \mathrm{mV}$. A-C. Time course of block by 0.2 or $10 \mu \mathrm{M} \alpha \mathrm{M}-\mathrm{MIIIJ}$ of EPSPs (A), MEPSPs (B), and ACh-evoked PSPs (C). The pair of asterisks along the top of panel B denotes the time interval flanked by the pair of asterisks in panel A. Insets in panels A and C show sample traces before (1) and in the presence of $0.2 \mu \mathrm{M} \alpha \mathrm{M}$-MIIIJ (2) or $10 \mu \mathrm{M} \alpha \mathrm{M}$-MIIIJ (3). Hump in falling phase of EPSPs in inset of panel A presumably reflects the multiple innervation of the fiber. Sample traces in panel B were obtained before (left) and in the presence of $0.2 \mu \mathrm{M} \alpha \mathrm{M}-\mathrm{MIIIJ}$ (right); each trace shows two MEPSPs, and the calibration scale applies to both traces. No MEPSPs were discernable in the presence of $10 \mu \mathrm{M}$ $\alpha \mathrm{M}$-MIIIJ. Early spike of superimposed traces in inset of $\mathrm{C}$ is an artifact of the iontophoretic pulse.

\subsection{Intracellular Recording of Synaptic Activity in Goldfish Intercostal (IC) Muscle}

In view of $\alpha$ M-MIIIJ's ability to paralyze goldfish as well as block nAChRs in X. laevis frog, we examined whether the peptide also blocked nAChR function in goldfish, whose intercostal (IC) muscle proved simple to dissect (see Section 5.6.). Spontaneous MEPSPs were readily observed regardless of the site of penetration of the intracellular recording electrode. This is because the muscle fibers are relatively short ( 1 mm) and have NMJs distributed all over their surface (see Figure S2B) as expected of fish fast muscles. $\alpha \mathrm{M}-\mathrm{MIIIJ}(1 \mu \mathrm{M})$ readily blocked spontaneous MEPSPs in goldfish IC muscles (Figure 5). 


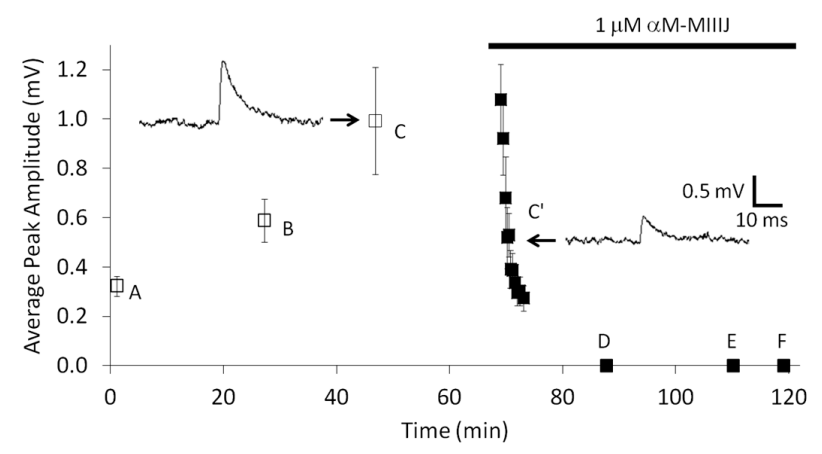

Figure 5. $\alpha \mathrm{M}$-MIIIJ $(10 \mu \mathrm{M})$ blocks MEPSPs in goldfish intercostal (IC) muscles. Intracellular recordings, performed as described in Methods, were obtained successively from six muscle fibers, A through F. Each data point represents the mean peak amplitude \pm SD of at least 30 events. Recordings were made before (fibers A-C) and during (fibers $C^{\prime}-\mathrm{F}$ ) exposure to $\alpha \mathrm{M}-\mathrm{MIII}(1 \mu \mathrm{M})$. After recordings from fiber $C$ were acquired, the bath was supplemented with a high concentration of $\alpha \mathrm{M}-\mathrm{MIIIJ}$ such that its final concentration in the bath was $1 \mu \mathrm{M}$, and the recording recommenced (fiber $\mathrm{C}^{\prime}$ ) until the resting potential was abruptly lost. The resting potentials (in $\mathrm{mV}$ ) of the respective fibers fibers were as follows: -67 (fiber A), -62 (fiber B), -92 (fiber C), -84 (fiber C'), -98 (fiber D), -65 (fiber E), and -101 (fiber F). In fibers D-F, no discernable MEPSPs were observed above the noise and indicated the block by $1 \mu \mathrm{M}$ $\alpha \mathrm{M}-\mathrm{MIIIJ}$ was $>80 \%$ in each case.

aM-MIIIJ clearly blocks the ACh-mediated postsynaptic responses in both X. laevis and goldfish muscles. The block was concentration dependent as illustrated in Figure 6, which combines the results from different preparations. In the figure, there are also three binding curves for a simple bimolecular interaction of toxin and receptor with an $\mathrm{IC}_{50}$ of $0.05,0.1$ or $0.2 \mu \mathrm{M}$ (see Figure 6 legend for equation). The potencies of $\alpha \mathrm{M}-\mathrm{MIIIJ}$ in blocking $\mathrm{nACh}$ receptors of $\mathrm{X}$. laevis and goldfish muscles were similar, with an $\mathrm{IC}_{50}$ estimated to be within about a factor of two of $0.1 \mu \mathrm{M}$.

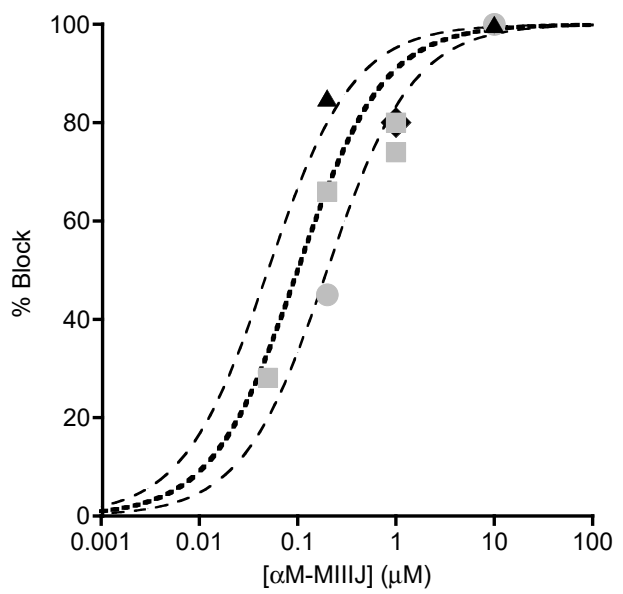

Figure 6. Concentration-dependent block by $\alpha \mathrm{M}-\mathrm{MIIIJ}$ of synaptically- and ACh-evoked responses at frog and fish NMJs. Plot summarizing results with different concentrations of $\alpha \mathrm{M}-\mathrm{MIIIJ}$ from experiments of the sort illustrated in Figure 4; Figure 5. Percentage block of: EPSPs in X. laevis LP (circles, 2); spontaneous MEPSPs in X. laevis LP (squares, 4) and goldfish IC (diamond, which denotes the minimum block of $80 \%$ by $1 \mu \mathrm{M}$ peptide in fibers D, E, and F in Figure 5); and ACh-evoked responses in X. laevis LP (triangles, 2). Each point represents one measurement. Block of EPSPs were not corrected for non-linear summation [24] and are therefore minimum estimates. Lines are curves of the Langmuir adsorption isotherm, \% Block $=100 \% /\left\{1+\left(\mathrm{IC}_{50} /[\right.\right.$ Toxin] $\left.)\right\}$, where [Toxin] is $\alpha \mathrm{M}-\mathrm{MIIIJ}$ concentration, and $\mathrm{IC}_{50}=0.1,0.05$ or $0.2 \mu \mathrm{M}$ (central bold-dotted line and flanking dashed lines, respectively). Experimental points lie close to or between the dashed lines and suggest an aggregate $\mathrm{IC}_{50}$ within a factor of about two of $0.1 \mu \mathrm{M}$. 


\section{6. $\alpha$ M-MIIIJ Reversibly Blocks ACh-Gated Currents $\left(I_{A C h}\right)$ of Zebrafish-Muscle nAChRs Exogenously} Expressed in X. laevis Oocytes

Three combinations of $\mathrm{nAChRs}$ subunits expressed in zebrafish muscle have been identified: $\alpha \beta \delta \gamma$ (embryonic fibers), $\alpha \beta \delta \varepsilon$ (larval and adult fast fibers), and $\alpha \beta \delta$ (slow fibers) [25] (see also [26,27]). The susceptibilities of these nAChRs to $\alpha \mathrm{M}-\mathrm{MIIIJ}$ were examined in two-electrode voltage-clamped oocytes expressing each of the three combinations of zebrafish subunits, and the ACh-gated currents $\left(\mathrm{I}_{\mathrm{ACh}}\right)$ were measured to monitor the function of the nAChRs. $\alpha \mathrm{M}-\mathrm{MIIIJ}$ blocked the $\mathrm{I}_{\mathrm{ACh}}$ of all three subunit combinations with $\mathrm{IC}_{50} \mathrm{~s}$ in the range of $50 \mathrm{nM}$ (Figure 7, $\mathrm{IC}_{50}$ values are in Table 2). These results are consistent with what we observed with $\mathrm{nAChRs}$ endogenously expressed in muscles of Xenopus frog and goldfish (Figure 6).

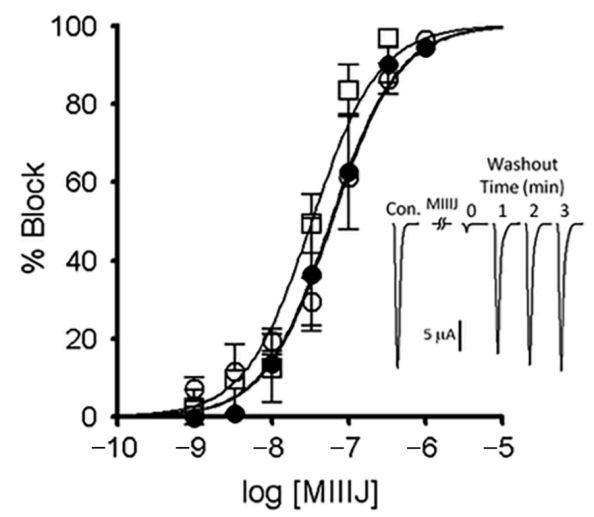

Figure 7. $\alpha \mathrm{M}-\mathrm{MIIIJ}$ blocks ACh-gated currents $\left(\mathrm{I}_{\mathrm{ACh}}\right)$ of voltage-clamped X. laevis oocytes exogenously expressing zebrafish nAChRs. Expression of nAChRs, induction of $\mathrm{I}_{\mathrm{ACh}}$ in voltage-clamped oocytes, and toxin application and analysis of consequent effects were performed as described in Methods. Concentration-dependent block by $\alpha \mathrm{M}$-MIIIJ of three combinations of zebrafish $\mathrm{nAChR}$ subunits are plotted ( $\alpha \beta \delta \varepsilon$, closed circles; $\alpha \beta \delta \gamma$, open circles; and $\alpha \beta \delta$, open squares). Each data point represents mean $\pm \mathrm{SE}$ ( $n=3$ or 4 oocytes). Solid line represents best-fit curve of the data to the Langmuir adsorption isotherm (see Table 2 for $\mathrm{IC}_{50} \mathrm{~s}$ ). Inset illustrates example $\mathrm{I}_{\mathrm{ACh}}$ traces from an oocyte expressing the $\alpha \beta \delta$-subunit combination before (control), immediately after a 5-min. exposure to $330 \mathrm{nM} \alpha \mathrm{M}-\mathrm{MIIJ}$, and following peptide washout at 1-min. intervals; duration of each trace is $4 \mathrm{sec}$. The block by $\alpha \mathrm{M}-\mathrm{MIIIJ}$ was readily reversible.

Table 2. $\mathrm{IC}_{50} \mathrm{~s}$ of $\alpha \mathrm{M}-\mathrm{MIIIJ}$ and $\alpha$-EI on nAChRs expressed in X. laevis oocytes. ${ }^{1}$.

\begin{tabular}{|c|c|c|}
\hline $\mathrm{nAChR}^{2}$ & $\alpha \mathrm{M}-\mathrm{MIIIJ}$ & $\alpha$-EI \\
\hline$z \alpha \beta \delta$ & $0.033(0.023-0.048)^{3}$ & $0.073(0.045-0.118)^{4}$ \\
\hline$z \alpha \beta \delta \gamma$ & $0.061(0.049-0.076)^{3}$ & $0.103(0.069-0.155)^{4}$ \\
\hline$z \alpha \beta \delta \varepsilon$ & $0.058(0.040-0.085)^{3}$ & $0.058(0.039-0.087)^{4}$ \\
\hline$z \alpha \beta \varepsilon$ & $0.034(0.022-0.053)^{5}$ & N. A. \\
\hline$m \alpha \beta \delta \gamma$ & $44.4(25.4-77.5)^{6}$ & N.A. \\
\hline$m \alpha \beta \delta \varepsilon$ & $3.6(2.6-4.9)^{6}$ & N. A. \\
\hline$h \alpha \beta \delta \gamma$ & $19.6(13.8-27.7)^{6}$ & N. A. ${ }^{7}$ \\
\hline$h \alpha \beta \delta \varepsilon$ & $4.1(2.9-5.9)^{6}$ & N. A. \\
\hline $\mathrm{h} \alpha 9 \alpha 10$ & $>>10^{8}$ & N. A. \\
\hline $\mathrm{r} \alpha 4 \beta 2$ & $>>10^{8}$ & N. A. ${ }^{9}$ \\
\hline $\mathrm{r} \alpha 3 \beta 4$ & $>>10^{8}$ & N. A. ${ }^{10}$ \\
\hline
\end{tabular}

${ }^{1} \mathrm{IC}_{50}$ value in $\mu \mathrm{M}, 95 \%$ C.I. in parentheses. N.A. indicates not tested or not available (but see related information in enumerated footnote). ${ }^{2}$ Prefix: $z$, zebrafish; $m$, mouse; $h$, human; $r$, rat. Greek letters: combination of $n A C h R$-subunit cRNAs injected into a given oocyte. ${ }^{3}$ from Figure 7. ${ }^{4}$ see Figure $9 .{ }^{5}$ see Figure 8 A. ${ }^{6}$ see Figure $11 .{ }^{7} \mathrm{IC}_{50}=0.187$ $\pm 0.043 \mu \mathrm{M}$ (mean \pm S.E.) from patch-clamped human rhabdomyocarcoma cell line TE671 expressing h $\alpha \beta \gamma \delta$ [28]. ${ }^{8}$ No block observed with $10 \mu \mathrm{M}$, the highest $\alpha \mathrm{M}$-MIIIJ concentration tested $\left(n=3\right.$ oocytes). ${ }^{9} \mathrm{I}_{\mathrm{ACh}}$ in patch-clamped HEK293 line expressing h $\alpha 4 \beta 2$ nAChRs was $60 \%$ blocked by $10 \mu \mathrm{M} \alpha$-EI [28]. ${ }^{10} \mathrm{I}_{\mathrm{ACh}}$ in patch-clamped HEK293 line expressing $\mathrm{h} \alpha 3 \beta 4 \mathrm{nAChRs}$ was $90 \%$ blocked by $10 \mu \mathrm{M} \alpha$-EI [28]. 
nAChRs comprised of only three different subunit combinations ( $\alpha \beta \gamma, \alpha \beta \delta$, and $\alpha \beta \varepsilon$ receptors) are not endogenously expressed except in zebrafish, which express $\alpha \beta \delta$ nAChRs as described above. Such combinations of mammalian $n A C h R$ subunits have been exogenously expressed in X. laevis oocytes to help identify the binding sites of competitive nAChR antagonists (e.g., [29]). Toward that end, we also tried to functionally express zebrafish $\alpha \beta \gamma$ - and $\alpha \beta \varepsilon$-receptor combinations in oocytes. Thus far, we have successfully expressed only the latter and characterized its block by $\alpha \mathrm{M}$-MIIIJ (Figure 8A,B).
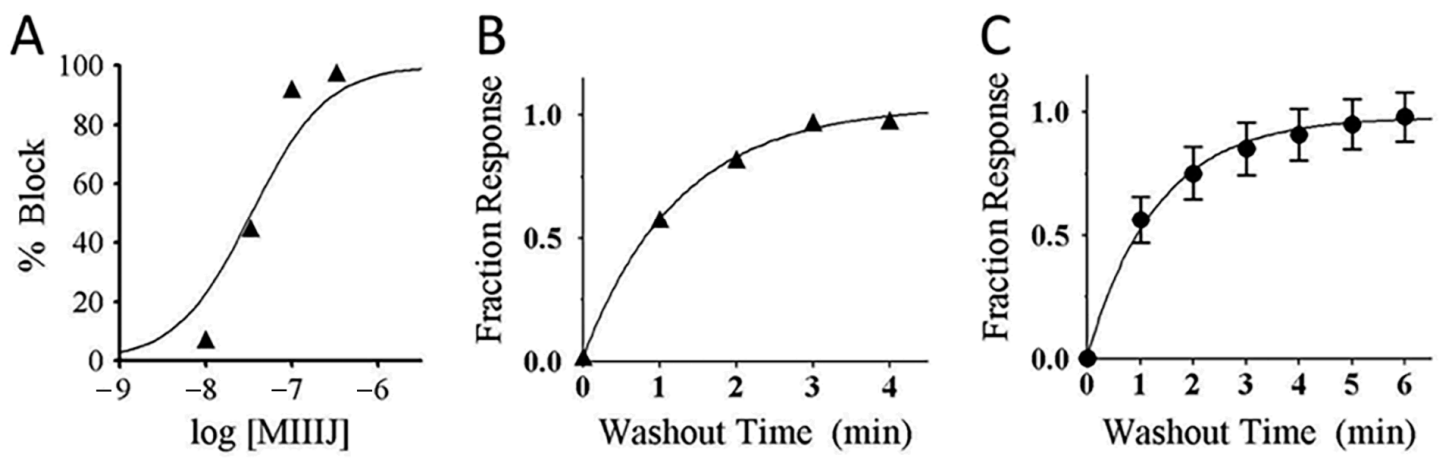

Figure 8. $\alpha$ M-MIIIA block of $\alpha \beta \varepsilon$ nAChR (A), and washout kinetics from $\alpha \beta \varepsilon$ AChR (B) and $\alpha \beta \delta \varepsilon$ $\mathrm{nAChR}(\mathrm{C})$. These experiments were done essentially as in Figure 7. A. $\alpha \mathrm{M}-\mathrm{MIII}$ concentration-response curve for block of $\alpha \beta \varepsilon \mathrm{nAChR}$ (see Table 2 for $\mathrm{IC}_{50}$ ). The data points follow a trajectory steeper than their fit to a Langmuir isotherm (solid curve), the significance of which remains to be determined. B, C. $\alpha$ M-MIIIJ washout curves from $\alpha \beta \varepsilon$ (B) and $\alpha \beta \delta \varepsilon$ (C) nAChRs. Solid line is the best-fit single-exponential curve with a $\mathrm{k}_{\text {off }}$ of $0.80 \mathrm{~min}^{-1}$ (95\% C.I. of $\left.0.7-0.9\right)$ for $\alpha \beta \varepsilon$, and $0.76 \mathrm{~min}^{-1}(95 \%$ C.I. of 0.4-1.2) for $\alpha \beta \delta \varepsilon$. Data points represent mean \pm S.E. $(n=3$ for panels $\mathbf{A}$ and $\mathbf{B}$; and $n=6$ for panel $\mathbf{C}$, from aggregate of open circles in Figure 9B,E below).

The reversibility of $\alpha \mathrm{M}$-MIIIJ's block of $\mathrm{I}_{\mathrm{ACh}}$ during its washout from $\alpha \beta \delta \varepsilon$ and $\alpha \beta \varepsilon$ nAChRs was relatively slow ( $\mathrm{k}_{\text {off }}$ of $0.80 \mathrm{~min}^{-1}$ (95\% C.I. of $0.7-0.9$ ) for $\alpha \beta \varepsilon$, and $0.76 \mathrm{~min}^{-1}$ (95\% C.I. of $0.4-1.2$ ) for $\alpha \beta \delta \varepsilon$, Figure $8 \mathrm{~B}, \mathrm{C})$. For subunit combinations lacking the $\varepsilon$-subunit $(\alpha \beta \delta$ and $\alpha \beta \delta \gamma)$, however, $\mathrm{I}_{\mathrm{ACh}}$ recovered almost completely within a minute of $\alpha \mathrm{M}$-MIIIJ washout (e.g., Figure 7 inset) with a $\mathrm{k}_{\text {off }}$ of $2.4 \mathrm{~min}^{-1}$ (95\% C.I. of 0.0-5.2) for $\alpha \beta \delta \gamma$, and $2.5 \mathrm{~min}^{-1}$ (95\% C.I. of 1.1-3.9) for $\alpha \beta \delta$ (data were the aggregate of open circles in Figure 9A,D and C,F, respectively). 

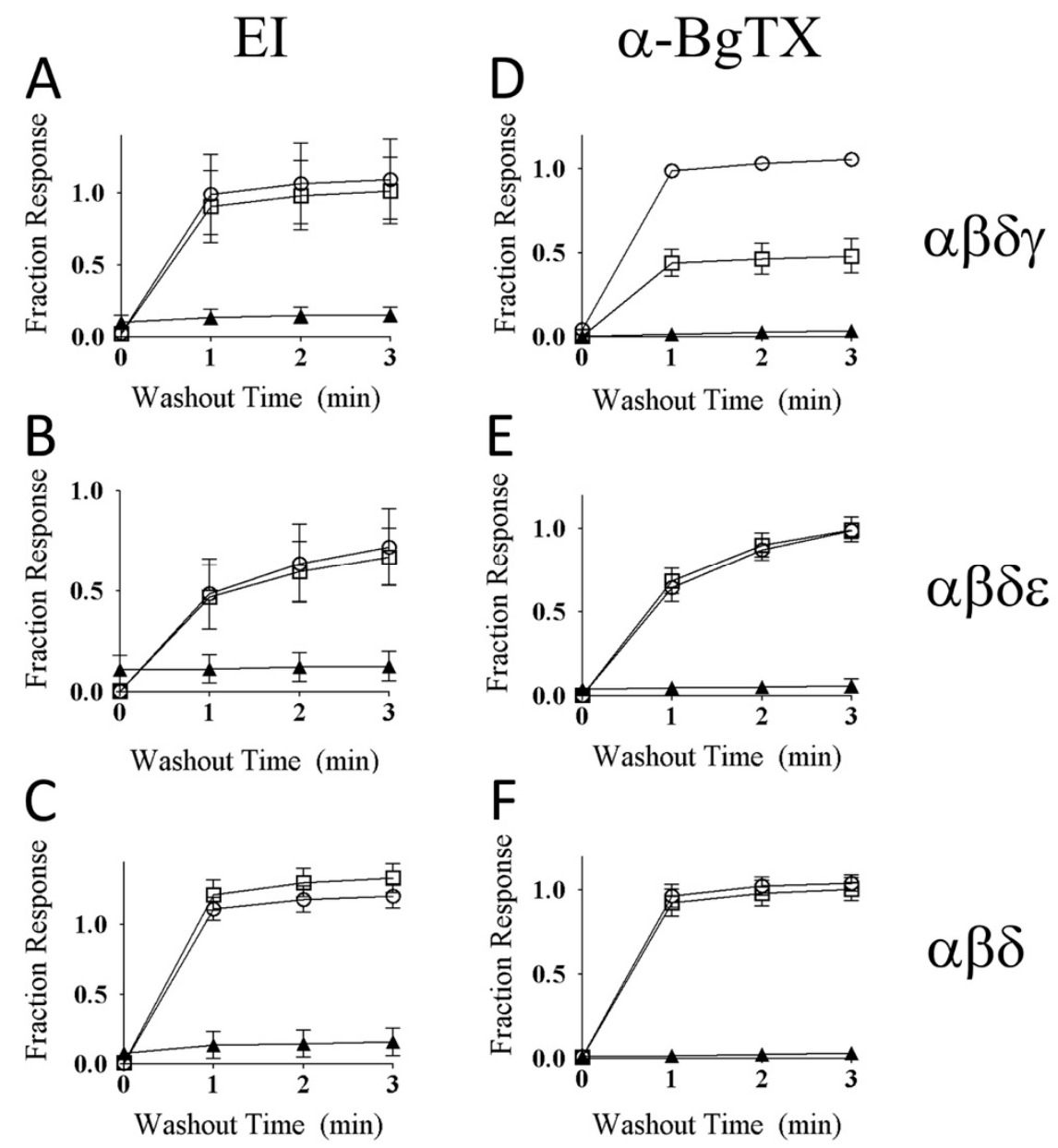

Figure 9. $\alpha \mathrm{M}$-MIIIJ $(10 \mu \mathrm{M})$ protects zebrafish $\mathrm{nAChRs}$ against slowly-reversible block by $\alpha$-EI and $\alpha$-bungarotoxin ( $\alpha$-BgTX). ACh-gated currents from oocytes expressing zebrafish nAChRs were obtained as described in Methods. A given oocyte was first exposed to $10 \mu \mathrm{M} \alpha \mathrm{M}-\mathrm{MIIIJ}$ for $10 \mathrm{~min}$. in a static bath, then perfused to observe the time course of recovery from block (open circles). The oocyte was then exposed again to $10 \mu \mathrm{M} \alpha \mathrm{M}-\mathrm{MIIIJ}$ for $5 \mathrm{~min}$. in a static bath, after which the bath was supplemented with either $1 \mu \mathrm{M} \alpha$-EI (open squares in panels $\mathrm{A}, \mathrm{B}$, and C) or $10 \mu \mathrm{g} / \mathrm{mL} \alpha$-BgTX (open squares in panels D, E, and F) and allowed to sit for another 5 min before the bath perfusion was recommenced. Finally, in a separate experiment, a given oocyte was exposed to either $1 \mu \mathrm{M} \alpha$-EI alone (panels A, B, and C) or $10 \mu \mathrm{g} / \mathrm{mL}$ of $\alpha$-BgTX alone (panels D, E, and F) for 5-min in a static bath before bath perfusion was recommenced (solid triangles). The time courses of recovery from block during the perfusion following the static-bath exposure to toxin(s) are plotted. Recovery from block following exposure to $\alpha$-EI alone or $\alpha$-BgTX alone was very slow in all panels (solid triangles). Furthermore, in all instances, except panel D, pre- and concurrent exposure to $\alpha \mathrm{M}$-MIIIJ prevented persistent block by $\alpha$-EI and $\alpha$-BgTX (as evident from similarity of time courses curves denoted by open circles and open squares). The divergent open-circle and open-square curves in panel D indicate that $\alpha \mathrm{M}-\mathrm{MIIIJ}$ only partially protected the $\alpha \beta \delta \gamma$ receptor against block by $\alpha$-BgTX. $\alpha$-BgTX use in these experiments was derivatized with tetramethylrhodamine (same stock solution as that used in fluorescence imaging experiments described below).

\section{7. $\alpha M$-MIIIJ Protects Zebrafish nAChRs Against A-Bungarotoxin ( $\alpha$-BgTX) and A-Conotoxin EI ( $\alpha$-EI)}

To examine the mechanism by which $\alpha$ M-MIIIJ blocks the zebrafish nAChRs, we performed competition-blocking experiments between $\alpha \mathrm{M}-\mathrm{MIIIJ}$ and two other $\mathrm{nAChR}$ antagonists: $\alpha$-BgTX and $\alpha$-EI. $\mathrm{I}_{\mathrm{ACh}}$ of all three subunit combinations were irreversibly blocked by $\alpha$-BgTX (see Figure 9D-F) and slowly-reversibly blocked by $\alpha$-EI (Figure 10 and its inset, also see Figure 9A-C). 


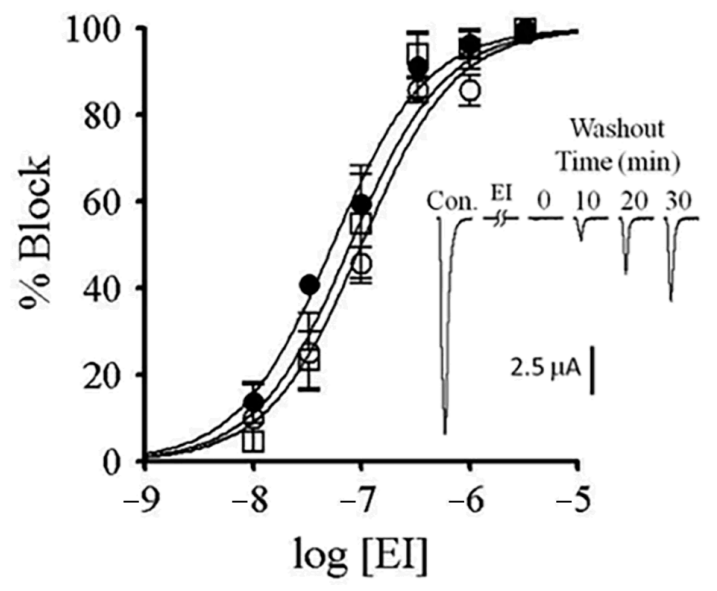

Figure 10. Block by $\alpha$-conotoxin EI of zebrafish muscle $n A C h R s$ expressed in X. laevis oocytes. The block of $\mathrm{I}_{\mathrm{ACh}}$ by conotoxin was assessed as described in Figure 7. Plotted are the concentration-response curves for the block by $\alpha$-EI of $\alpha \beta \delta$ (open squares), $\alpha \beta \delta \gamma$ (open circles), and $\alpha \beta \delta \varepsilon$ (closed circles) nAChRs. Each data point represents mean \pm S.E. $(n=3-5)$. (See Table 2 for $\mathrm{IC}_{50}$ s.) Inset shows example $\mathrm{I}_{\mathrm{ACh}}$ traces from an oocyte expressing $\alpha \beta \delta \varepsilon \mathrm{nAChRs}$ before and following a 5-min. exposure to $1 \mu \mathrm{M}$ $\alpha$-EI; duration of each trace is $5 \mathrm{~s}$. The block by $\alpha$-EI is only slowly reversible.

When oocytes were exposed first to $10 \mu \mathrm{M} \alpha \mathrm{M}$-MIIIJ for 5-min., followed by a 5-min exposure to a mixture of $10 \mu \mathrm{M} \alpha \mathrm{M}$-MIIIJ and $1 \mu \mathrm{M} \alpha$-EI, the block of $\mathrm{I}_{\mathrm{ACh}}$ for all three subunit combinations of zebrafish $n A C h R s$ tested $(\alpha \beta \gamma \delta, \alpha \beta \delta \varepsilon$, and $\alpha \beta \delta)$ was rapidly reversed upon toxin washout (compare circles and squares in Figure 9A-C). The same was observed when $\alpha$-BgTX $(10 \mu \mathrm{g} / \mathrm{mL})$ was used instead of $\alpha$-EI with $\alpha \beta \delta$ and $\alpha \beta \delta \varepsilon$ nAChRs (Figure 9E,F), which indicates that these two nAChRs were largely protected by $\alpha \mathrm{M}$-MIIIJ against block by $\alpha$-BgTX. However, for $\alpha \beta \delta \gamma \mathrm{nAChRs,} \alpha \mathrm{M}$-MIIIJ provided only partial protection against $\alpha$-BgTX (Figure 9D).

2.8. $\alpha$ M-MIIIJ Blocks Indirectly-Evoked Mouse Muscle Action Potentials and Exogenously-Expressed Mouse and Human Muscle $n A C h R s$

As described above, $\alpha \mathrm{M}-\mathrm{MIIIJ}$ failed to paralyze mice in in-vivo assays, and we wondered whether this was a consequence of inadequate dosage or possible pharmacokinetic effects. Thus, we tested the peptide on an isolated mouse muscle preparation and observed that $10 \mu \mathrm{M} \alpha \mathrm{M}-\mathrm{MIIIJ}$ blocked muscle action potentials evoked by motor-nerve stimulation (Figure 11). To more quantitatively assess the pharmacology of $\alpha \mathrm{M}-\mathrm{MIIIJ}$, we tested the peptide on mammalian muscle nAChRs exogenously-expressed in X. laevis oocytes. At micromolar concentrations, $\alpha \mathrm{M}-\mathrm{MIIIJ}$ blocked $\mathrm{I}_{\mathrm{ACh}}$ of $\alpha \beta \delta \gamma$ and $\alpha \beta \delta \varepsilon$ nAChRs from both mouse and human (Figure 12). We conclude that $\alpha \mathrm{M}-\mathrm{MIIIJ}$ blocks both endogenous and exogenously-expressed mammalian muscle nAChRs, albeit with considerably less potency than muscle nAChRs of fish, the natural prey of $C$. magus. 


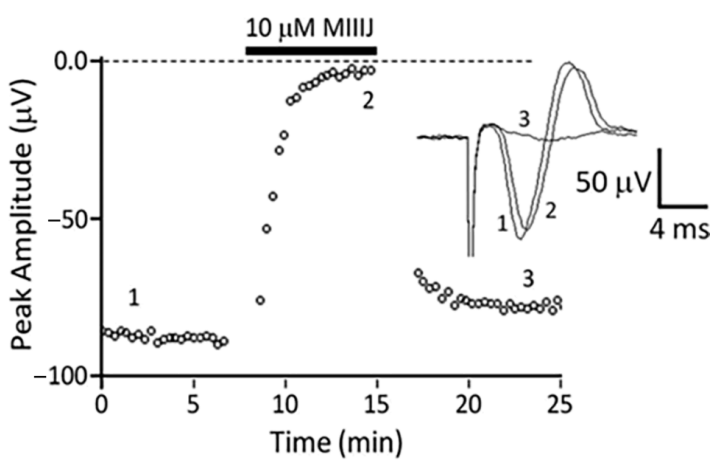

Figure 11. $\alpha \mathrm{M}$-MIIIJ $(10 \mu \mathrm{M})$ blocks neuromuscular transmission in mouse muscle. Extracellular recording of the mouse levator auris longus (LAL) muscle's action potential evoked by indirect stimulation was performed as described in Methods. Plot of peak (negative) amplitude of muscle action potential as a function of time before, during exposure to $10 \mu \mathrm{M} \alpha \mathrm{M}-\mathrm{MIIIJ}$ (indicated by black bar), and after toxin washout. Inset shows example traces of the extracellularly-recorded action potential before (trace 1), during block by toxin (trace 2) and after washout (trace 3), with trace numbers corresponding to times indicated in the main plot. The results are consistent with a low-potency block of mouse muscle nAChRs; direct evidence for this is illustrated in Figure 12.
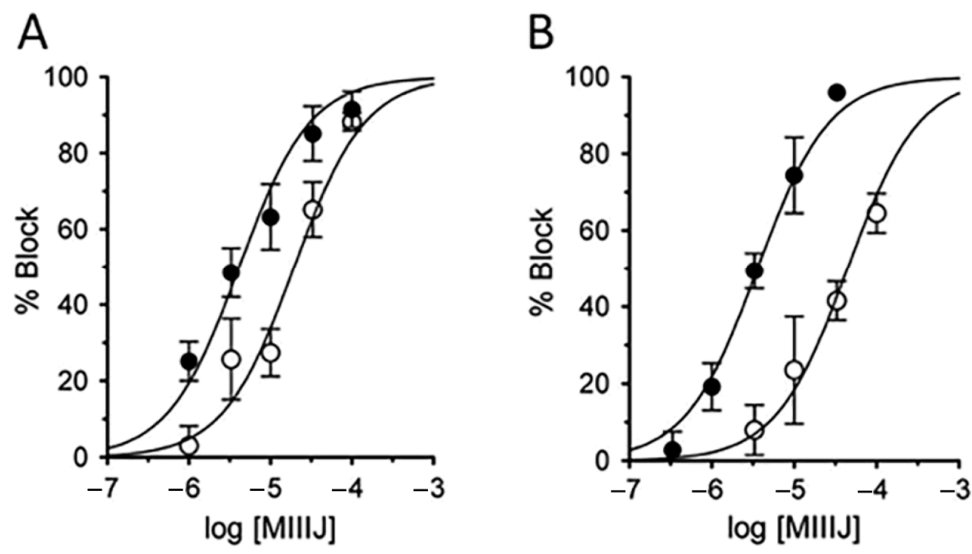

Figure 12. $\alpha \mathrm{M}-\mathrm{MIIIJ}$ blocks mammalian muscle $\mathrm{nAChRs}$ with low potencies. Oocytes expressing $n A C h R s$ from human (A) and mouse (B) were voltage-clamped and exposed to $\alpha \mathrm{M}$-MIIIJ as described in Methods. Concentration-response curves for the block by $\alpha \mathrm{M}-\mathrm{MIIIJ}$ of $\mathrm{I}_{\mathrm{ACh}}$ in oocytes expressing $\alpha \beta \delta \gamma$ (open circles) or $\alpha \beta \delta \varepsilon$ (closed circles) subunits. Solid lines represent best-fit curves to the Langmuir isotherm (see Table 2 for $\mathrm{IC}_{50} \mathrm{~s}$ ).

\subsection{Lack of Effects of $\alpha M-M I I I J$ on Neuronal nAChRs}

To examine $\alpha \mathrm{M}-\mathrm{MIIIJ}$ 's selectivity for muscle over neuronal nAChRs, we tested $\alpha \mathrm{M}-\mathrm{MIIIJ}$ on the following exogenously-expressed neuronal nAChRs: $\alpha 3 \beta 4$ (rat), $\alpha 4 \beta 2$ (rat), $\alpha 9 \alpha 10$ (human). $\alpha$ M-MIIIJ $\left(10 \mu \mathrm{M}\right.$, the highest concentration tested) failed to block $\mathrm{I}_{\mathrm{ACh}}$ of any of these receptors (Table 2).

\subsection{Imaging the Binding of Fluorescently-Labeled $\alpha$-BgTX at Frog NMJs}

To further investigate $\alpha \mathrm{M}$-MIIIJ's mechanism of action, we examined the manner in which the peptide affected $\alpha$-BgTX binding. Tetramethylrhodamine conjugated $\alpha$-bungarotoxin (TMR $\alpha$-BgTX) readily stained NMJs of both X. laevis LP and R. pipiens CP muscles (Figure 13C,F, respectively). Muscles were pre-treated with fluorescein peanut agglutinin (f-PNA) so the progress of TMR $\alpha$-BgTX binding at a given NMJ could be tracked (Figure 13B,E). In control experiments (i.e., in the absence of $\alpha \mathrm{M}-\mathrm{MIIIJ}$ ), the binding of $1 \mu \mathrm{g} / \mathrm{mL}$ TMR $\alpha$-BgTX to the X. laevis NMJ leveled off within about 20 min (Figure 13A, muscle I, squares). Similar results were obtained with R. pipiens CP preparations (cf., Figure 13F). 

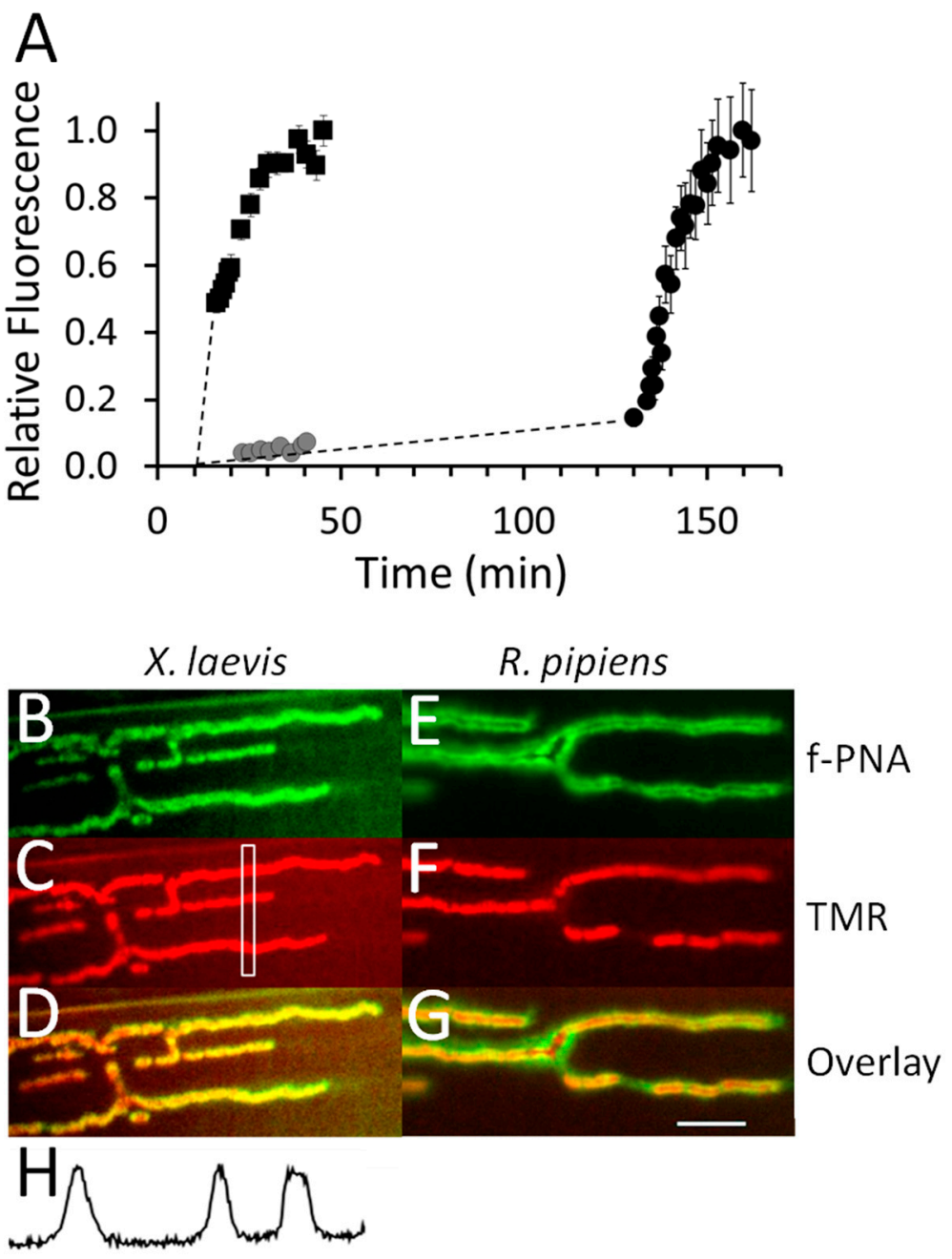

Figure 13. $\alpha \mathrm{M}-\mathrm{MIIIJ}(10 \mu \mathrm{M})$ inhibits binding of TMR $\alpha$-BgTX at neuromuscular junctions of X. laevis LP muscle. Preparation of muscles, image acquisition, and quantification of fluorescence were performed as described in Methods. A. Two preparations, Muscle I and Muscle II, were stained with f-PNA so locations of NMJs could be identified as described in Methods. In Muscle I, the NFR bathing the muscle was replaced (at $t=10^{\prime}$ ) with $1 \mu \mathrm{g} / \mathrm{mL}$ TMR $\alpha$-BgTX, and the NMJ was periodically imaged while its staining ensued until a steady state was achieved (squares). Muscle II was treated with $10 \mu \mathrm{M} \alpha \mathrm{M}-\mathrm{MIIIJ}$ (at $t=0$ ) for 10-min. before it was exposed to $1 \mu \mathrm{g} / \mathrm{mL}$ TMR $\alpha$-BgTX $+10 \mu \mathrm{M} \alpha \mathrm{M}-\mathrm{MIIIJ}$, after which the endplate was periodically imaged for 30-min. (gray circles). Then the solution bathing muscle II was removed and reserved, and the muscle rinsed. After $\sim 90-\mathrm{min} .$, Muscle II was exposed to $1 \mu \mathrm{g} / \mathrm{mL}$ TMR $\alpha$-BgTX alone while the NMJ was periodically imaged until the fluorescence reached steady state (black circles). The steady-state level of fluorescence varied from one NMJ to the next, so the fluorescence values at each endplate were normalized to the value obtained at steady state (at $t \approx 30^{\prime}$ for Muscle I and $t \approx 140^{\prime}$ for Muscle II). Each data point represents the mean \pm SD from 9 to 12 regions of interest (ROIs; see rectangle in panel C) obtained along the NMJ. Presence of $\alpha \mathrm{M}-\mathrm{MIIIJ}$ clearly interfered with $\alpha$-BgTX binding. B and E. f-PNA staining of an NMJ from X. laevis LP muscle (B) and R. pipiens CP muscle (E). C and F. TMR $\alpha$-BgTX staining of X. laevis LP muscle (C) and R. pipiens CP muscle (F). Here, the CP muscle was treated with the reserved TMR $\alpha$-BgTX $+\alpha M$-MIII solution removed from Muscle II; nevertheless, strong TMR staining is clearly evident. D and G. Overlays of images in panels B and C (D) and panels E and F (G). H. Fluorescence profile from the ROI in panel C (see rectangle therein). Calibration bar ( $20 \mu \mathrm{m}$, white line) in G applies to panels B through G. Presence of $\alpha \mathrm{M}$-MIIIJ clearly inhibited the binding of TMR $\alpha$-BgTX at the NMJ of the X. laevis LP muscle but not at that of the $R$. pipiens CP muscle. 
In contrast, when $10 \mu \mathrm{M} \alpha \mathrm{M}$-MIIIJ was present, very little binding of TMR $\alpha$-BgTX to the X. laevis NMJ was observed even after 30 min (Figure 13A, muscle II, gray circles). Muscle II was washed for $>$ $60 \mathrm{~min}$. (to allow the $\alpha \mathrm{M}$-MIIIJ to unbind) and then exposed to TMR $\alpha$-BgTX alone. This time, TMR $\alpha$-BgTX readily bound and reached steady state in about $20 \mathrm{~min}$ (Figure 13A, muscle II, black circles), just as was observed for muscle I (Figure 13A, squares).

As a positive control, the 'used' mixture of $\alpha$-BgTX $+\alpha$ M-MIIIJ to which muscle II had been exposed was applied to a 'naïve' $R$. pipiens CP preparation for about 10-min and imaged. The $R$. pipiens NMJs were robustly stained by TMR $\alpha$-BgTX (Figure 13F), consistent with our results indicating that synaptic responses of $R$. pipiens CP muscles, unlike those of X. laevis LP muscles, are relatively resistant to block by $\alpha \mathrm{M}-\mathrm{MIIIJ}$ (Figure 3B). More to the point, these results also showed that: a) $\alpha \mathrm{M}$-MIIIJ had not somehow inactivated the $\alpha$-BgTX to produce the protection represented by the gray circles in Figure 13A; and b) $\alpha$ M-MIIIJ's association with nAChRs is reversible, consistent with electrophysiological results (e.g., Figure 2C, Figure 7 inset, Figures 8 and 9).

\subsection{Imaging the Binding of Fluorescently-Labeled $\alpha$-BgTX at NMJs of Goldfish IC Muscle}

We next examined whether $\alpha \mathrm{M}$-MIIIJ interfered with the binding of TMR $\alpha$-BgTX to goldfish NMJs. We found that f-PNA could not be used as a marker for goldfish NMJs; so, goldfish IC muscle preparations were treated in two ways. Treatments involved exposure to TMR $\alpha$-BgTX $(1 \mu \mathrm{g} / \mathrm{mL})$ for $10 \mathrm{~min}$ in the presence of $\alpha \mathrm{M}-\mathrm{MIIIJ}(10 \mu \mathrm{M}$, Treatment I) or in the absence of $\alpha \mathrm{M}$-MIIIJ (Treatment II). Preparations were then rinsed and imaged. Minimal TMR $\alpha$-BgTX staining was observed in the presence of $\alpha$ M-MIIIJ (Figure S2A, Treatment I). On the other hand, robust staining was observed in the absence of the conotoxin (Figure S2A, Treatment II). These results demonstrate that, just as with $X$. laevis LP muscles, $\alpha \mathrm{M}-\mathrm{MIII}$ protects goldfish IC muscles against $\alpha$-BgTX binding.

\subsection{Binding of Fluorescently-Labeled $\alpha$-BgTX at Zebrafish NMJs}

Goldfish intercostal muscle fibers have NMJs distributed over their entire surface (Figure S2B) and are therefore presumably fast muscles. To confirm our functional results, obtained with exogenously-expressed zebrafish fast and slow muscle nAChRs, on endogenous channels at the level of toxin binding, we exploited the fact that while NMJs of fast fibers are widely distributed, NMJs of slow fibers are largely confined to their myoseptal ends [30]. Toward this end, we performed competition-binding studies with fluorescently-labeled $\alpha$-BgTX on a preparation from larval zebrafish, whose skin is relatively transparent and whose muscles have both fast and slow fibers, as described in Methods (see also Figure S3B).

This experiment employed Alexa488 $\alpha$-BgTX (which fluoresces green) in addition to TMR $\alpha$-BgTX, and it was performed in three phases. In phase 1, the preparation was lightly treated with TMR $\alpha$-BgTX to provide an irreversible marker for NMJs. In the subsequent two phases, the preparation was treated with Alexa488 $\alpha$-BgTX, first in the presence of $\alpha \mathrm{M}$-MIIIJ (phase 2), then in the absence of $\alpha \mathrm{M}$-MIIIJ (phase 3), with washing after each phase followed by imaging. About an hour elapsed between the end of phase 2 and the start of phase 3. The mean values of green (Alexa488 $\alpha$-BgTX) fluorescence at NMJs of both fast and slow fibers were lower after Phase 2 than after Phase 3, showing that $\alpha \mathrm{M}-\mathrm{MIIIJ}$ $(10 \mu \mathrm{M})$ inhibited $\alpha$-BgTX binding to both types of muscle fibers (Figure S3A).

Taken together, the results of our electrophysiological and fluorescence-imaging experiments demonstrate that $\alpha \mathrm{M}-\mathrm{MIIIJ}$ avidly targets nAChRs at the NMJs of frog (X. laevis) and fish (C. auratus and D. rerio).

\section{Discussion}

\section{1. $\alpha M$-MIIIJ Uncovers a New Family of Muscle nAChR-Targeting Conotoxins}

$\alpha \mathrm{M}-\mathrm{MIIIJ}$ and related sequences from other fish-hunters form a new class within the large M-superfamily of conotoxins. M-superfamily toxins are highly diverse and are found in nearly all cone 
snail species studied to date where they are known to exert various functions [21]. Two well-studied classes within the M-superfamily are $\mu$-conotoxins which target voltage-gated Na channels [31] and $\psi$-conotoxins which block muscle $\mathrm{nAChR}$ without competing with the bungarotoxin binding site [20]. While these toxins all belong to the M-superfamily and share the same cysteine framework with $\alpha \mathrm{M}-\mathrm{MIIIJ}$, their primary amino acid sequences are highly divergent (Figure 1A, bottom alignments). In contrast, $\alpha \mathrm{M}-\mathrm{MIIIJ}$ and related sequences from the Pionoconus clade are highly conserved and share a number of identical residues (Figure 1A, black arrowheads above top alignment). We demonstrate here that $\alpha \mathrm{M}-\mathrm{MIIIJ}$ blocks muscle $\mathrm{nAChR}$ and propose that the $\alpha \mathrm{M}-\mathrm{MIIIJ}-$-like peptides are also antagonists of muscle nAChR. Future functional studies are needed to investigate this hypothesis.

\section{2. $\alpha M-M I I I J$ Blocks Muscle nAChRs of X. laevis Frogs}

Three ion-channel targets of known paralytic conotoxins are presynaptic V-gated Ca channels, postsynaptic nAChRs, and skeletal muscle V-gated Na channels (Table 1). With regard to X. laevis muscle, $\alpha$ M-MIIIJ did not block directly-evoked action potentials (Figure 2B), thus ruling out muscle $\mathrm{Na}$ channels as a target of the peptide. During exposure to $\alpha \mathrm{M}-\mathrm{MIIIJ}$, no indication of quantized block (Figure 4A) or sparing of MEPSPs (Figure 4B) was observed, unlike the effects of $\omega$-GVIA, which blocks presynaptic Ca channels at the frog NMJ [32]. Likewise, during the exposure to $\alpha \mathrm{M}-\mathrm{MIIIJ}$, the block of EPSPs progressed smoothly with no major decrements (Figure 4A) that would suggest conduction failure in the motor axon; thus, we conclude that the peptide did not block action potentials (and by extension, V-gated Na channels) in the nerve. In contrast, $\alpha \mathrm{M}$-MIIIJ blocked the muscle's response to ionotophoretically- (i.e., exogenously-) applied ACh (Figure 4C), clearly demonstrating that $\alpha \mathrm{M}-\mathrm{MIIIJ}$ is an antagonist of nAChRs.

\section{3. $\alpha M$-MIIIJ Blocks nAChRs of Goldfish Muscle}

$\alpha \mathrm{M}$-MIIIJ is from a fish-hunting cone (see Introduction). Thus, the observation that the peptide paralyzes goldfish (Table S1) was not unexpected. With the knowledge that nAChRs were a target of $\alpha \mathrm{M}-\mathrm{MIIIJ}$, we next focused our attention on nAChRs of goldfish muscle. $\alpha \mathrm{M}-\mathrm{MIIIJ}$ clearly blocked spontaneous miniature synaptic potentials in goldfish intercostal muscles (Figure 5).

\section{4. $\alpha M$-MIIIJ Blocks $I_{A C h}$ of Zebrafish-Muscle nAChRs Exogenously Expressed in X. laevis Oocytes}

The nAChR subunit compositions of fast and slow muscles of both larval and older zebrafish have been identified as $\alpha \beta \delta \varepsilon$ and $\alpha \beta \delta$, respectively [25]. $\alpha$ M-MIIIJ blocked nAChRs composed of both subunit combinations (Figure 7). For good measure, we also tested the embryonic $(\alpha \beta \delta \gamma)$ combination and found it was also blocked by $\alpha \mathrm{M}-\mathrm{MIIIJ}$ (Figure 7). All three subunit combinations yielded $\alpha \mathrm{M}-\mathrm{MIIIJ}$ concentration-response curves with similar $\mathrm{IC}_{50}$ s and slopes (Figure 7), with the $\alpha \beta \delta$ combination having an affinity about a factor of two higher than that of the other two (Table 2). The $\alpha \beta \varepsilon$-subunit combination would be expected to have a subunit-stoichiometry for the pentameric nAChR of $\alpha_{2} \beta_{1} \varepsilon_{2}$, with two $\alpha / \varepsilon$ interfaces, while the $\alpha \beta \delta \varepsilon$-combination would have one $\alpha / \varepsilon$ interface (and one $\alpha / \delta$ interface). The $\mathrm{IC}_{50}$ of $\alpha \mathrm{M}-\mathrm{MIIIJ}$ for $\alpha \beta \varepsilon$ was similar to that for $\alpha \beta \delta$ (Table 2). It might be noted that for a receptor with two binding sites with identical affinity for a toxin, the $\mathrm{IC}_{50}$ for functional block of the receptor would be the square-root of the affinity constant of the sites [33]. These results suggest that $\alpha$ M-MIIIJ binds with about equal avidity to both the $\alpha / \delta$ and $\alpha / \varepsilon$ interfaces, which is consistent with our $\alpha$ BgTX-competition experiments (Figure 9E,F). $\alpha$ M-MIIIJ provided $\alpha \beta \delta \gamma$ nAChRs only-partial protection against $\alpha$-BgTX (Figure 9D), suggesting that $\alpha \mathrm{M}$-MIIIJ had a lower affinity for the $\alpha / \gamma$-interface than the $\alpha / \delta$ - and $\alpha / \varepsilon$-interfaces; alternatively, $\alpha$-BgTX bound to the $\alpha / \gamma$ interface more quickly than to either the $\alpha / \delta$ - or $\alpha / \varepsilon$-interfaces. This issue may be resolved when functional expression of $\alpha \beta \gamma$ nAChRs is achieved. 


\section{5. $\alpha M-M I I J$ Inhibits the Binding of $\alpha$-BgTX to Frog and Fish NMJs}

Fluorescently-tagged $\alpha$-BgTX has long served as an extremely useful marker for nAChRs since the initial synthesis of TMR $\alpha$-BgTX and its use to stain R. pipiens and X. laevis NMJs [34]. We observed that $\alpha \mathrm{M}$-MIIIJ inhibited the binding of $\alpha$-BgTX at the NMJs of X. laevis (Figure 13A), goldfish (Figure S2A) and zebrafish (Figure S3A). As might be expected, $\alpha$ M-MIIIJ did not interfere with the binding of $\alpha$-BgTX at the NMJ of R. pipiens (Figure 13F).

It is intriguing that $\alpha \mathrm{M}-\mathrm{MIIIJ}$ blocks muscle $\mathrm{nAChRs}$ of $X$. laevis but not those of R. pipiens. This result is consistent with our observations that $\alpha \mathrm{M}-\mathrm{MIIIJ}$ protects nAChRs of X. laevis (Figure 13A), but not those of R. pipiens (Figure 13F), against $\alpha$-BgTX. It would be interesting to explore the underpinnings of the difference at the molecular level. It might be noted that neuronal nAChRs of closely-related frogs can have different sequences, as was observed where the driving force for variation involved resistance to epibatidine, a nAChR-targeting neurotoxic alkaloid used by some frogs for chemical defense [35].

\subsection{Possible Rationale for the Presence of both a-MI and $\alpha M-M I I J$ in C. magus Venom}

Aside from $\alpha$-MI and $\alpha$ M-MIIIJ, each of the C. magus venom peptides listed in Table 1 has a distinct ion-channel target. $\alpha$-MII was much more potent than $\alpha$-MI in blocking synaptic transmission in sympathetic ganglia of frog, which led us to speculate that the role of $\alpha$-MII in C. magus venom is to interfere with the flight-or-fight response of fish prey [36], in contrast to the role of $\alpha$-MI, which is to immobilize fish prey.

$\alpha$-MI is potent in paralyzing mice, and in view of its sequence homology to $\alpha$-GI, $\alpha$-GIA and $\alpha$-GII (Table 3), which block muscle nAChRs [37], $\alpha$-MI was presumed to also block muscle nAChRs [8]. Indeed, competition-binding experiments with $\left[{ }^{125} \mathrm{I}\right] \alpha$-BgTX revealed that $\alpha$-MI blocks muscle nAChRs by binding with high affinity to the $\alpha / \gamma$ (of Torpedo) and $\alpha / \delta$ (of mouse) ACh-binding sites of the receptor [22,38]. $\alpha$-MIC, the most recently characterized $\alpha$-MI congener, has a similar $\mathrm{LD}_{50}$ against fish (Xiphophorous helleri) as that of $\alpha$-MI; furthermore, $\alpha$-MIC competitively inhibits the binding of $\left[{ }^{3} \mathrm{H}\right]$ epibatidine to the muscle subtype of $\mathrm{nAChRs}$ from Torpedo californica with a $\mathrm{K}_{\mathrm{i}}$ similar to that of $\alpha$-MI [39].

Table 3. Peptides of fish-hunting Conus that block nAChRs. ${ }^{1 .}$ Cysteine are in bold

\begin{tabular}{|c|c|c|}
\hline Peptide $^{2}$ & Sequence $^{3}$ & Reference \\
\hline \multicolumn{3}{|l|}{$\alpha(3 / 5)$} \\
\hline$\alpha$-GI & ECCNPACGRHYSC\# & Gray 1981 [37] \\
\hline$\alpha$-GIA & ECCNPACGRHYSCGK\# & Gray 1981 [37] \\
\hline$\alpha-G I B$ & ECCNPACGRHYSCKG\# & McIntosh 2002 [40] \\
\hline$\alpha-G I I$ & ECCHPACGKHFSC\# & Gray $1981[37]$ \\
\hline$\alpha-\mathrm{MI}$ & GRCCHPACGKNYSC\# & McIntosh 1982 [8] \\
\hline$\alpha$-MIA & DGRCCHPACAKHFNC\# & McIntosh 2002 [40] \\
\hline$\alpha-\mathrm{MIB}$ & NGRCCHPACGKNYSC\# & McIntosh 2002 [40] \\
\hline$\alpha-\mathrm{MIC}$ & CCHPACGKNYSC\# & Kapono 2013 [39] \\
\hline$\alpha-S I$ & ICCNPACGPKYSC\# & Zafaralla 1988 [41] \\
\hline$\alpha$-SIA & YCCHPACGKNFDC\# & Myers 1991 [42] \\
\hline$\alpha-S I I$ & GCCCNPACGPNYGCGTSCS & Ramilo 1992 [43] \\
\hline$\alpha-A c 1.1 \mathrm{a}$ & NGRCCHPACGKHFNC\# & Liu 2007 [44] \\
\hline$\alpha-A c 1.1 b$ & NGRCCHPACGKHFSC\# & Liu 2007 [44] \\
\hline$\alpha-\mathrm{CnIA}$ & GRCCHPACGKYYSC\# & Favreau 1999 [45] \\
\hline$\alpha-\mathrm{CnIB}$ & CCHPACGKYYSC\# & Favreau 1999 [45] \\
\hline \multicolumn{3}{|l|}{$\alpha(4 / 7)$} \\
\hline$\alpha-E I$ & RDOCCYHPTCNMSNPQIC\# & Martinez 1995 [22] \\
\hline$\alpha$-MII† & GCCSNPVCHLEHSNLC\# & Cartier 1996 [9] \\
\hline$\alpha-G I C+$ & GCCSHPACAGNNQHIC\# & McIntosh 2002 [40] \\
\hline$\alpha$-GID + & IRD $\gamma$ CCSNPACRVNNOHVC & Nicke 2003 [46] \\
\hline$\alpha$-PIAt & RDPCCSNPVCTVHNPQIC\# & Dowell 2003 [47] \\
\hline$\alpha$-PeIAt & GCCSHPACSVNHPELC\# & McIntosh 2005 [48] \\
\hline
\end{tabular}


Table 3. Peptides of fish-hunting Conus that block nAChRs. ${ }^{1}$ Cysteine are in bold

\begin{tabular}{|c|c|c|}
\hline Peptide $^{2}$ & Sequence $^{3}$ & Reference \\
\hline \multicolumn{3}{|l|}{$\alpha(4 / 4)$} \\
\hline$\alpha$-BuIAt & GCCSTPPCAVLYC\# & Azam 2005 [49] \\
\hline$\alpha-\mathrm{PIB}$ & ZSOGCCWNPACVKNRC\# & Lopez-Vera 2007 [50] \\
\hline$\alpha$-EIIA & ZTOGCCWNPACVKNRC\# & Quinton 2013 [51] \\
\hline \multicolumn{3}{|l|}{$\alpha \mathrm{A}_{\text {Long }}$} \\
\hline$\alpha A-P I V A$ & GCCGSYONAACHOCSCKDROSYCGQ\# & Hopkins 1995 [52] \\
\hline$\alpha A-E I V A$ & GCCGPYONAACHOCGCKVGROOYCDROSGG\# & Jacobsen 1997 [33] \\
\hline$\alpha \mathrm{A}-\mathrm{EIVB}$ & GCCGKYONAACHOCGCTVGROOYCDROSGG\# & Jacobsen 1997 [33] \\
\hline \multicolumn{3}{|c|}{ - } \\
\hline$\alpha$ A-OIVA & CCGVONAACHOCVCKNTC\# & Teichert 2004 [53] \\
\hline$\alpha \mathrm{A}-\mathrm{OIVB}$ & CCGVONAACPOCVCNKTCG\# & Teichert 2005b [54] \\
\hline $\begin{array}{l}\alpha \mathrm{A}-P e I V A \\
\alpha \mathrm{S}\end{array}$ & & Teichert 2006 [55] \\
\hline$\alpha S-R V I I A$ & KCNFDKCKGTGVYNCG $\gamma S C S C \gamma$ GLHSCRCTY & \\
\hline & NIGSMKSGCACICTYY & Teichert 2005a [54] \\
\hline \multirow{2}{*}{$\alpha$ S-GVIIIB +} & SGSTCTCFTSTNCQGSCECLSPPGCYCSNN & \\
\hline & GIRQRGCSCTCPGT\# & Christensen 2015 [56] \\
\hline \multicolumn{3}{|c|}{ (1) } \\
\hline $\begin{array}{l}\alpha \mathrm{C}-\operatorname{PrXA} \\
\alpha \mathrm{M}\end{array}$ & TYGIYDAKPOFSCAGLRGGCVLPONLROKFKE\# & Jimenez 2007 [29] \\
\hline$\psi$-PIIIE & HOOCCLYGKCRRYOGCSSASCCQR\# & Shon $1997[20]$ \\
\hline$\psi$-PIIIF & GOOCCLYGSCROFOGCYNALCCRK\# & Van Wagoner 2003 [57] \\
\hline$\psi-\operatorname{Pr} 3 e$ & AARCCTYHGSCLKEKCRRKYCC\# & Lluisma 2008 [18] \\
\hline$\alpha \mathrm{M}-\mathrm{MIIIJ}$ & ZKCCSGGSCPLYFRDRLICPCC & This report \\
\hline
\end{tabular}

The disparity between the structures of $\alpha$-MI and $\alpha \mathrm{M}-\mathrm{MIIIJ}$ raises the question as to whether $\alpha \mathrm{M}-\mathrm{MIIIJ}$ binds to the same site of muscle nAChRs as does $\alpha$-MI. Our results showing that $10 \mu \mathrm{M}$ $\alpha \mathrm{M}-\mathrm{MIIIJ}$ almost completely inhibits $\alpha$-BgTX binding (Figure 13A, Figures S2A and S3A) suggest that at this concentration $\alpha \mathrm{M}$-MIIIJ likely occludes both $\alpha$-BgTX-binding sites of a given $\mathrm{nAChR}$. These results are consistent with the protection $\alpha \mathrm{M}-\mathrm{MIIIJ}$ affords the zebrafish $\mathrm{nAChR}$ subunit combinations $\alpha \beta \delta$ (slow-fiber nAChRs) and $\alpha \beta \delta \varepsilon$ ('adult' fast fiber) against block of their $\mathrm{I}_{\mathrm{ACh}}$ by $\alpha$-BgTX (Figure $9 \mathrm{E}, \mathrm{F}$ ). The only-partial protection $10 \mu \mathrm{M} \alpha \mathrm{M}-\mathrm{MIIIJ}$ afforded the $\alpha \beta \delta \gamma \mathrm{nAChR}$ subunit combination (Figure 9D) suggests that $\alpha$-BgTX may bind more quickly to the $\alpha / \gamma$ interface than to either the $\alpha / \delta$ or $\alpha / \varepsilon$ interfaces. Alternatively, $\alpha$ M-MIIIJ may have a lower affinity for the $\alpha / \gamma$ interface than for either the $\alpha / \delta$ or $\alpha / \varepsilon$ interfaces. We did not perform any experiments on embryonic zebrafish muscle, which express $\alpha \beta \delta \gamma$ nAChRs. In view of our electrophysiological results of Figure 9D, we predict that $\alpha \mathrm{M}-\mathrm{MIIIJ}$ would only partially protect NMJs of embryonic muscle against staining by fluorescent $\alpha$-BgTX.

In the discussion above, we assumed that $\alpha \mathrm{M}$-MIIIJ interacts directly with each of the two ACh-binding (or orthosteric) sites of the receptor. A far less parsimonious possibility is that $\alpha \mathrm{M}-\mathrm{MIIIJ}$ modulates the ACh-binding sites of the receptor by binding at an allosteric site. The rationale for entertaining the latter possibility is to understand why C. magus venom has two structurally divergent peptides ( $\alpha \mathrm{MI}$ and $\alpha \mathrm{M}-\mathrm{MIIIJ})$ to accomplish the same physiological endpoint-incapacitation of the $\mathrm{nAChR}$. That is, perhaps $\alpha$-MI acts orthosterically while $\alpha \mathrm{M}-\mathrm{MIIIJ}$ acts allosterically. This issue may be resolved by experiments involving mutagenesis of specific residues in the $\mathrm{nAChR}$ subunits or by examining whether $\alpha \mathrm{MI}$ and $\alpha \mathrm{M}-\mathrm{MIIIJ}$ can act synergistically.

A species of fish-hunting Conus with three subfamilies of peptides that target muscle nAChRs is C. ermineus, whose venom contains $\alpha$-EI (an $\alpha-4 / 7$ subfamily member), $\alpha$ A-EIVA/B (two closely-related 
$\alpha$ A-subfamily peptides that differ in only two residues), and the more recently-discovered $\alpha$-EIIA (Table 3). $\alpha$-EI binds preferentially to the $\alpha \delta$ interface of mouse nAChRs whereas $\alpha A$-EIVA binds equally well to both the $\alpha \delta$ and $\alpha \gamma$ interfaces [33]. Our tests with $\alpha$-EI on zebrafish muscle nAChRs indicate that it blocks all three subunit combinations $(\alpha \beta \delta, \alpha \beta \delta \varepsilon$, and $\alpha \beta \delta \gamma)$ with similar potencies (Figure 10). Furthermore, $\alpha \mathrm{M}$-MIIIJ completely protected against the block by $\alpha$-EI of all three subunit combinations (Figure 9A-C). It remains to be seen which sites $\alpha \mathrm{A}$-EIVA binds to on zebrafish muscle nAChRs.

C. purpurascens is a fish-hunter with nAChR-targeting conotoxins belonging to three subfamilies: $\alpha$-PIB, $\alpha$ A-PIVA, and $\psi$-PIIIE/F (Table 3). $\psi$-PIIIE blocks $\mathrm{I}_{\mathrm{ACh}}$ of X. laevis oocytes expressing Torpedo nAChRs but does not compete with $\left[{ }^{125} \mathrm{I}\right] \alpha$-BgTX binding to Torpedo nAChRs [20]. Although the binding site of $\psi$-PIIIE remains to be identified, it is clear that $C$. purpurascens venom has subfamilies of conotoxins that block $n A C h R s$ via different modes or mechanisms. In this vein, it might be noted that $C$. imperialis, a worm-hunting species of Conus, has two closely related $\alpha$-conotoxins that block $\alpha 7$ nAChRs, $\alpha$-ImI and $\alpha$-ImII [58,59]. While $\alpha$-ImI competes with $\alpha$-BgTX, $\alpha$-ImII does not [58]. The binding of $\alpha$-ImII to $\alpha 7 \mathrm{nAChRs}$ has been investigated [60], and its binding site and mechanism of action were given the moniker "monkey wrench" [61].

It might be noted that there is the possibility that $\alpha \mathrm{M}-\mathrm{MIIIJ}$ may act promiscuously, and its more (or equally) important target in the wild is an ion channel other than muscle nAChRs; only further testing will resolve this issue. There is precedent for a conotoxin affecting the function of more than a single ion-channel target, for examples: $\mu \mathrm{O}$-conotoxin MrVIA/B can block both V-gated $\mathrm{Na}$ and V-gated $\mathrm{Ca}$ channels of snail neurons [62,63] (in addition to blocking vertebrate V-gated Na channels [64-66]); $\mu$-conotoxin CnIIIA, originally shown to block V-gated Na channels [12] can also block neuronal nAChRs [67]; $\mu$-conotoxins PIIIA and SIIIA, originally shown to block Na channels $[15,23]$ can also block $\mathrm{K}$ channels in the $\mathrm{K}_{\mathrm{V}} 1$ family [68]. Recently, an $\alpha$-conotoxin from C. eburneus, Eu1.6, was characterized that blocked (when tested at $1 \mu \mathrm{M}$ ) N-type voltage-gated Ca channels in dissociated mouse dorsal root ganglion neurons. Among various rat $\mathrm{nAChRs}$ exogenously expressed in $\mathrm{X}$. laevis oocytes, $10 \mu \mathrm{M}$ Eu1.6 blocked $\alpha 7 \mathrm{nAChRs}$ best (where $\mathrm{I}_{\mathrm{ACh}}$ was attenuated by about a half) but had no effect on skeletal muscle nAChRs with a subunit composition of $\alpha \beta \delta \varepsilon$ [69].

\section{Conclusions}

$\alpha \mathrm{M}-\mathrm{MIIIJ}$ blocks the function of nAChRs in the postsynaptic membrane of skeletal muscles of fish and frogs. The peptide also interferes with $\alpha$-BgTX binding to these receptors. It is well established that $\alpha$-BgTX blocks nAChR function by occluding the ACh-binding sites on the receptor; by extension, we conclude that $\alpha \mathrm{M}-\mathrm{MIIIJ}$ blocks nAChR function by interfering with ACh binding to nAChRs, although it remains to be seen by how similar a mechanism as that of $\alpha$-BgTX.

We reveal that $\alpha \mathrm{M}$-MIIIJ belongs to a new class of toxins from the M-superfamily and identify several toxin sequences with significant sequence homology to $\alpha \mathrm{M}$-MIIIJ. We hypothesize that these and additional homologs likely to be identified in the future will also function as muscle nAChR antagonists. These studies will expand our understanding of the biology of fish hunting and the versatility of toxins belonging to the M-superfamily, and further highlight the remarkable adaptability of toxin genes in these marine predators.

\section{Materials and Methods}

\section{1. $\alpha M-M I I I J$ Purification and Sequencing}

Crude venom was extracted from C. magus ( 200 specimens) collected in waters surrounding the island of Marinduque in the Philippines. Lyophilized venom was extracted with $1.1 \% \mathrm{v} / \mathrm{v}$ acetic acid and chromatographed on a column of Sephadex G25 $(2.5 \times 92 \mathrm{~cm})$ as previously described (McIntosh et al., 1982 [8], method 3). Low molecular mass fractions were subsequently separated by reversed-phased liquid chromatography (HPLC) on a Supelco LC18 semi-prep column (10 mm id $\times$ 
$25 \mathrm{~cm} ; 5 \mu \mathrm{m}$ particle size) at a flow rate of $3.0 \mathrm{~mL} / \mathrm{min}$. A $0.1 \%$ TFA- $0.01 \%$ TFA, $60 \%$ acetonitrile buffer system was used for elution. The gradient was $15 \% \mathrm{~B} \times 5 \mathrm{~min}$, increasing to $25 \% \mathrm{~B}$ at $25 \mathrm{~min}$ and increasing to $60 \% \mathrm{~B}$ at $35 \mathrm{~min}$. Absorbance was monitored at $210 \mathrm{~nm}$.

Peptide from the final purification was stored in the HPLC buffer in which it eluted. A $287-\mu \mathrm{L}$ solution of this purified peptide ( $250 \mathrm{pmol})$ was combined with $14.4 \mu \mathrm{L}(20: 1 \mathrm{v} / \mathrm{v})$ of $0.5 \mathrm{M}$ Tris base which raised the $\mathrm{pH}$ to a value between 7 and 8 as measured with $\mathrm{pH}$ paper. Seventy-five $\mu \mathrm{L}$ of $50 \mathrm{mM}$ dithiothreitol was added (final concentration $10 \mathrm{mM}$ ); the reaction vessel was flushed with argon, and the reaction incubated at $65{ }^{\circ} \mathrm{C}$ for $15 \mathrm{~min}$. The solution was allowed to cool; $15 \mu \mathrm{L}$ of $20 \% 4$-vinyl pyridine in ethanol was added, and the solution was reacted for a further $25 \mathrm{~min}$ at room temperature in the dark. The solution was diluted 3-fold with $0.1 \%$ trifluoroacetic acid, and the alkylated peptide was purified on the Brownlee column.

Sequencing was performed with Edman chemistry on an Applied Biosystems 477A (Applied Biosystems, Foster City, CA, USA) Protein Sequencer at the Protein/DNA Core Facility at the University of Utah Cancer Center (Salt Lake City, UT, USA). Mass spectrometry was performed as described previously [9].

Initial attempts at sequencing yielded no sequence suggesting that the $\mathrm{N}$-terminal residue might be pyroglutamate as found in previously characterized conotoxins. We therefore subjected the peptide to digestion with pyroglutamyl aminopepdtidease in an attempt to remove the potential pyroglutamate and allow sequencing.

An amount of $400 \mu \mathrm{g}$ of calf liver pyroglutamyl aminopepdtidease (Boehringer) enzyme powder ( $\sim 16 \mu \mathrm{g}$ protein) and $\sim 1 \mathrm{nmol}$ of $\alpha \mathrm{M}$-MIIIJ was dissolved in $100 \mu \mathrm{L}$ of incubation buffer consisting of $50 \mathrm{mM} \mathrm{Na} \mathrm{HPO}_{4}, \mathrm{pH} 7.3,10 \mathrm{mM} \beta$-mercaptoethanol, $1 \mathrm{mM}$ EDTA and incubated at $37^{\circ} \mathrm{C}$ for $30 \mathrm{~min}$. The reaction products were then purified by HPLC using a Vydac C18 $4.6 \mathrm{~mm}$ ID column using buffer $\mathrm{A}=0.1 \%$ TFA and buffer $\mathrm{B}=0.1 \%$ TFA, $60 \%$ acetonitrile buffer. The gradient was $20 \%$ buffer B increasing to $50 \%$ buffer B over $30 \mathrm{~min}$ The resulting product was successfully sequenced.

\subsection{Identification of Homologous Sequences}

Using the mature toxin sequence of $\alpha \mathrm{M}$-MIIIJ we searched for homologous sequences in C. magus and additional taser-and-tether hunting species from the Pionoconus clade (Conus consors and Conus striatus) in published venom gland transcriptomes $[14,17,70]$ using the Basic Local Alignment Search Tool (BLAST) [71] with an e-value of $<1 \times 10^{-1}$.

\section{3. $\alpha M-M I I I J$ Synthesis}

The peptide was manually assembled by solid-phase approach [72]. The starting Boc-Cys(Mob)$\mathrm{CM}$ resin with a capacity of $0.3 \mathrm{mmol} / \mathrm{g}$ was obtained according to published procedures [73]. All $\mathrm{N} \alpha$-tert-butyloxycarbonyl (BOC) protected amino acids with side chain protection were purchased from Bachem Inc. (Torrance, CA, USA). The side chain protecting groups were as follows: $\operatorname{Arg}(\operatorname{Tos})$, Asp(OChx), Cys(Mob), Lys(e-2ClZ), Ser(Bzl), Tyr(2BrZ). Z in the sequence represents pyroglutamic acid.

Three equivalents of Boc-amino acids based on the original substitution of the resin were used for each coupling. The couplings of the protected amino acids were achieved by adding three equivalents coupling reagents BOP (Benzotriazole-1-yl-oxy-tris(dimethylamino)-phosphoniumhexafluorophosphate) or HBTU (O-(benzotriazol-1-yl)- $N, N, N^{\prime}, N^{\prime}$-tetramethyluronium hexafluorophosphate) in dichloromethane and dimethylformamide, respectively to the resin and adjusting the $\mathrm{pH}$ to 9 with diisopropylethylamine (DIPEA) then shaking it for $20 \mathrm{~min}$. Completions of the couplings were monitored by the qualitative ninhydrin test [74]. Boc removal was achieved by treatment with $60 \%$ trifluoroacetic acid and 3\% ethanedithiol in $\mathrm{CH}_{2} \mathrm{Cl}_{2}$ for $30 \mathrm{~min}$. An isopropyl alcohol (containing $1 \%$ ethanedithiol) wash followed the TFA treatment and then successive washes with triethylamine solution $\left(10 \%\right.$ in $\mathrm{CH}_{2} \mathrm{Cl}_{2}$ ), methanol, triethylamine solution, methanol and $\mathrm{CH}_{2} \mathrm{Cl}_{2}$ completed the neutralization. The completed peptide was cleaved from the resin support with simultaneous side chain deprotection by using anhydrous hydrogen fluoride containing the scavengers anisole $(10 \% \mathrm{v} / \mathrm{v})$ 
and methyl sulfide $(1 \% \mathrm{v} / \mathrm{v})$ at $0{ }^{\circ} \mathrm{C}$ for $75 \mathrm{~min}$. The diethyl ether-precipitated crude peptide was extracted from the resin with 50\% isopropanol/ 50\% water solution, the $\mathrm{pH}$ was adjusted to $8-8.5$ with DIPEA and the mixture was slowly stirred for several days to complete the cyclization that was monitored by the Ellman test [75]. The peptide was purified by preparative HPLC. The cartridge used was hand packed, in house, with Waters polyethylene sleeve and frits and reversed-phase $300 \AA$ Vydac $C_{18}$ silica (15-20 $\mu \mathrm{m}$ particle size). The peptide was eluted with the solvent system $0.1 \%$ $\mathrm{TFA} / \mathrm{H}_{2} \mathrm{O} / \mathrm{CH}_{3} \mathrm{CN}$ at a flow rate of $100 \mathrm{~mL} / \mathrm{min}$. A linear gradient $1.5 \% \mathrm{~B}$ per 1 min increases from the baseline $\% \mathrm{~B}$ (Eluent $\mathrm{A}=0.1 \% \mathrm{TFA} / \mathrm{H}_{2} \mathrm{O}$, eluent $\mathrm{B}=60 \% \mathrm{CH}_{3} \mathrm{CN}, 40 \% \mathrm{~A}$ ) was applied. Capillary zone electrophoresis (CZE) was carried out on a Beckman P/ACE System 2050 controlled by an IBM Personal System/2 Model 50Z connected to a ChromJet integrator. CZE analysis employed a field strength of $10-20 \mathrm{kV}$ at $30{ }^{\circ} \mathrm{C}$ with a buffer of $15 \% \mathrm{CH}_{3} \mathrm{CN} / 85 \% 100 \mathrm{mM}$ sodium phosphate pH 2.5 on a Beckman eCAP capillary (363 mm o.d. $75 \mathrm{~mm}$ i.d. $50 \mathrm{~cm}$ length), LSIMS mass spectra were obtained with a JEOL JMS-HX110 double-focusing mass spectrometer (JEOL, Tokyo, Japan) fitted with a Cs+ gun.

The experiments described in this report employed synthetic $\alpha \mathrm{M}-\mathrm{MIIIJ}$.

\subsection{Animal Use}

Use of all animals in this study followed protocols approved by the University of Utah Institutional Animal Care and Use Committee (IACUC) that conform to the National Institutes of Health Guide for the Care and Use of Laboratory Animals. IACUC protocols were as follows: \#14-08018 (approved period, 20 August 2014-19 August 2017) and \#17-07020 (approved period, 01 August 2017-31 July 2020). Mice were euthanized with $\mathrm{CO}_{2}$. X. leavis and fish were anesthetized with Tricaine $(0.4 \%$ for $X$. laevis and goldfish, $0.02 \%$ for zebrafish) and decapitated. $R$. pipiens were sacrificed by double pithing.

\subsection{In Vivo Bioassays of $\alpha M-M I I I J$ on Goldfish and Mice}

Carassius auratus (goldfish). Toxin was dissolved in $0.9 \% \mathrm{w} / \mathrm{v} \mathrm{NaCl}(\mathrm{NSS})$, and $10 \mu \mathrm{L}$ were injected into the dorsal epaxial muscle of a given fish. The fish was then placed in a beaker $(14.5 \mathrm{~cm} \mathrm{dia.} \times$ $7.5 \mathrm{~cm}$ height) containing $700 \mathrm{~mL}$ of water with a 35-mm long, 10-mm diameter magnetic stir bar rotating at 200 RPM creating a vortex, such that the fish must actively swim to avoid being trapped in the center. Each of three control fish injected with NSS alone were able to swim with no sign of being overpowered by the vortex for $>35 \mathrm{~min}$. Fish injected with $\alpha \mathrm{M}-\mathrm{MIIIJ}$ gradually became paralyzed within several minutes and were unable to escape the vortex. After paralysis, fish were moved to $250 \mathrm{~mL}$ beakers to be observed. Motion of the mouth and gills was observed, but fish were unable to swim and did not respond to prodding of fins, dorsal and ventral sides, and eyes.

Mice. Swiss Webster mice ( $\sim 13 \mathrm{~g}$ avg. weight) were injected intraperitoneally (i.p.) with $\sim 2.3 \mathrm{nmol}$ $\alpha \mathrm{M}-\mathrm{MIIIJ} /$ gram mouse dissolved in $30 \mu \mathrm{L}$ NSS. Control mice were injected with $30 \mu \mathrm{L}$ NSS only. After injection, mice were observed for $>1.5 \mathrm{~h}$ for signs of paralysis.

\subsection{Isolated Muscle Preparations}

Rana pipiens (grass frog). The cutaneous pectoralis (aka cutaneus pectoris, CP) muscle of Rana pipiens ( $\sim 65 \mathrm{~mm}$ ) long adults of both sexes) was used. This flat, thin muscle has long served as a go-to preparation for studies of the structure and function of frog neuromuscular synapses (see e.g., [34,76,77]). For extracellular recording, the muscle was prepared and used as previously described [78]. For fluorescence microscopy, the preparation was pinned with 0.1 or $0.2 \mathrm{~mm}$ dia. stainless steel Minutien pins to coverslips (22 mm dia.) coated with Sylgard (Dow Corning, Midland, MI, USA), a transparent silicone elastomer.

Xenopus laevis (African clawed frog). These frogs do not have CP muscles, therefore we used their longitudinal pectoralis (LP) muscles, which are flat muscles that lie laterally on either side of the rectus abdominus muscle of the animal [79]. The motor nerve contacts the muscle near the rostral end and sends branches that run parallel with the muscle fibers. For extracellular recording, strips of LP muscles of adult animals were used. A given strip was placed in a multi-well chamber fabricated 
from Sylgard where the wells, each 4-mm diameter and 4-mm deep, were in a linear array with a $1 \mathrm{~mm}$-partition separating one well from the next. The rostral end of the strip was placed in well 1 and the caudal end in well 5 to 8 , depending on the strip's length. The portion of the muscle strip overlying a partition was covered with Vaseline. A pair of stimulating electrodes were located in wells 1 and 2 or wells 2 and 3; with a ground electrode located in well 3 in the former case, and in well 4 in the latter. A pair of recording electrodes were located in adjacent wells 4 and 5,5 and 6,6 and 7, or 7 and 8 (depending on muscle length). To obtain indirectly-evoked muscle action potentials without confounding, directly-evoked muscle action potentials, $10 \mu \mathrm{M} \mu$-conotoxin PIIIA, which specifically blocks the muscle subtype of V-gated $\mathrm{Na}$ channels [23], was present in the two wells in which the stimulating electrodes were located; thus, only the motor nerve was directly activated by the electrical stimulus. In a separate set of experiments, muscle action potentials in response to direct stimulation were recorded by having $10 \mu \mathrm{M}$ d-tubocurare in all wells to block synaptically-mediated responses. Excitatory postsynaptic responses evoked by indirect stimulation were recorded by placing $10 \mu \mathrm{M}$ $\mu$-conotoxin PIIIA in all wells.

For intracellular recording and fluorescence microscopy, we used LP muscles from $\sim 5$-cm juveniles of either sex. Cutting the muscle longitudinally approximately in half, a procedure that did not denervate the majority of the fibers in the medial half, allowed the remaining muscle to fit in a $\sim 23-\mathrm{mm}$ diameter, $\sim 2-\mathrm{mm}$ deep recording chamber or be pinned onto a Sylgard-coated coverslip (22 mm dia.).

Carassius auratus (goldfish). We discovered that the intercostal (IC) muscles of adult goldfish can be easily prepared for electrophysiology and are readily amenable for fluorescence microscopy following vital staining. Briefly, goldfish ( 4-cm long) of undetermined sex were anesthetized with $0.4 \%$ Tricaine, decapitated, and its skin descaled by plucking with serrated forceps. A pair of sharp-tipped scissors was used to make a longitudinal incision in the body wall along the ventral midline from anal fin to pelvic fin, with care taken to avoid cutting any entrails. This was followed, on each side of the animal, by two vertical incisions in the body wall from the ventral midline, dorsally to the spine: one from the anal fin and the other from the pelvic fin. Finally, each half of a rib cage, with its skin intact, was cut along the spine to free each hemi-rib cage from the rest of the animal. Each hemi-rib cage was pinned, skin-side down, in a Sylgard-coated plastic culture dish containing NFR, and the iridophore-laden parietal peritoneal integument overlying the inner surface of the ribs was carefully stripped away manually with tweezers to expose the two diagonally-overlapping layers of intercostal muscles. The intercostal muscle preparation, consisting of at least four ribs, was then pinned skin-side down to a Sylgard-coated coverslip (22-mm diameter). Two such preparations can be readily obtained from each side of a given goldfish.

Danio rerio (zebrafish). Briefly, larval zebrafish (4- to 6-day old), anesthetized with $0.02 \%$ Tricaine and decapitated, were pinned on their sides to a Sylgard-coated coverslip (15-mm dia.) with electrolytically-sharpened tungsten pins [80] essentially as described by Brehm's laboratory [81]. The skin on the uppermost side of each fish was then peeled away so toxins could readily access the underlying body musculature [81,82]. The transparency of the skin in these larval fish allowed the muscle fibers closest to the coverslip to be readily imaged with an inverted microscope. Slow fibers are distinguished by $\mathrm{nAChRs}$ localized largely to the myoseptal regions (at the ends of the muscle fibers), while fast muscles are invested with $\mathrm{nAChRs}$ distributed in clusters or puncta dispersed throughout a given muscle fiber (for review see [30]).

Mice. The levator auris longus (LAL) muscle initially described for electrophysiology by Faille and coworkers was used [83]. This thin, flat muscle preparation, which allowed ready access and egress of drugs, was pinned in a rectangular trough $(\sim 5 \times 15 \times 4 \mathrm{~mm}$ deep $)$ and the motor nerve was draped across two adjacent wells ( $\sim 4 \mathrm{~mm}$ dia. $\times 4 \mathrm{~mm}$ deep) and into the muscle-containing trough (all partitions were $1 \mathrm{~mm}$ wide and they, along with the overlying nerve, were covered with Vaseline). The chamber was fabricated from Sylgard.

Solutions. Both frog and fish muscle preparations were bathed in normal frog Ringer's (NFR), which consisted of (in $\mathrm{mM}$ ): $\mathrm{NaCl}$ (111); $\mathrm{KCl}$ (2); $\mathrm{CaCl}_{2}$ (1.8); and half-Na HEPES (10 mM), pH 7.2. 
Mouse muscle preparations were bathed in normal mammalian Ringer's (NMR) composed of (in mM): $\mathrm{NaCl}$ (145); $\mathrm{KCl}$ (5); $\mathrm{CaCl}_{2}$ (2); sodium citrate (1); glucose (10); and half-Na HEPES (10 mM), pH 7.4.

\subsection{Muscle Electrophysiology}

Extracellular recordings were acquired with a pair of wire electrodes connected to a Grass P55 A.C. preamplifier at an amplification of 100 or 1000 and bandpass filtered with $-6 \mathrm{~dB}$ cut-off frequencies of $1 \mathrm{~Hz}$ (unless otherwise indicated) and $1 \mathrm{kHz}$. The output of the preamp was digitized at a sampling rate of $10 \mathrm{kHz}$ with an NI PCI-6221 data acquisition board (National Instruments, Austin, TX, USA). Motor-nerve stimulation was accomplished with either a pair of wire electrodes, one each in the nerve-containing wells (R. pipiens $\mathrm{CP}$, adult X. laevis LP, mouse LAL preparations) or a suction electrode (juvenile $X$. laevis LP preparation). The stimulating electrodes were connected to a Grass Stimulus Isolation Unit ("SIU5A") driven by a Grass S88 stimulator (Grass Instruments, Quincy, MA, USA) that was set to deliver a supermaximal stimulus $(\sim 10 \mathrm{~V}, 0.1-\mathrm{ms}$ rectangular pulse) at a frequency of $1 / 20 \mathrm{sec}$. Responses were recorded with a pair of extracellular recording electrodes in the trough: one electrode located near the middle of the muscle, and the other near the caudal end of the muscle. A ground electrode was located in the trough between the stimulating and recording electrodes. All extracellular electrodes were stainless steel. For all electrophysiological recordings, timing of stimuli, and data acquisition, storage and retrieval were performed with home-made software written with LabVIEW (National Instruments, Austin, TX, USA).

Intracellular recordings were acquired with glass microelectrodes filled with $3 \mathrm{M} \mathrm{KCl}$ (X. laevis LP muscle) or $4 \mathrm{M}$ K-acetate (goldfish IC muscle) ( $~ 5 \mathrm{M} \Omega$ resistance) and a MultiClamp 700B amplifier (Molecular Devices, Sunnyvale, CA, USA) in current-clamp mode with current clamped at zero pA, where membrane potentials were amplified 10 -fold, high-frequency filtered at $1 \mathrm{kHz}$. (Note, $\mathrm{KCl}$ is the conventional electrolyte used to fill intracellular microelectrodes for recording from frog skeletal muscle (e.g., ref. [84]). Although K-acetate-filled microlectrodes are less popular, they have been used to record from goldfish skeletal muscle (see e.g., [85] We used $3 \mathrm{M} \mathrm{KCl}$ and $4 \mathrm{M} \mathrm{K}$-acetate solutions because we happened to have these on hand.) The output of the amplifier was digitized and synchronized with the stimulator using the hardware and software described above for extracellular recording. Insertion of the intracellular electrode at a location near an NMJ of an X. laevis LP fiber was assisted by use of fluorescein peanut agglutinin (f-PNA, see Section 5.9 below). NMJs of goldfish IC fibers are distributed all along a given fiber, so spontaneous miniature excitatory potentials (MEPSPs) were readily recorded regardless of electrode placement. Amplitudes of MEPSPs were measured manually.

Acetylcholine $(\mathrm{ACh})$ iontophoresis was performed in a manner similar to that previously described [84]. In essence, a Grass Instruments S-44 stimulator in conjunction with a home-made stimulus isolation unit (SIU) was used. One output of the SIU led to one terminal of "brake box" consisting of a $9-\mathrm{V}$ battery whose output was regulated by a potentiometer. The other terminal of the brake box was connected to a $100 \mathrm{M} \Omega$ current-limiting resistor located close to, and in series with, a chlorided silver wire that was inserted into a glass micropipette filled with $1 \mathrm{M} \mathrm{ACh \bullet Cl}$ and whose resistance was about $10 \mathrm{M} \Omega$. The other output of the SIU let to a chlorided silver wire bath electrode that provided the return path for ACh current. A (negative) braking current of $\sim 15 \mathrm{nA}$ was used to prevent desensitization-inducing ACh from leaking out of the pipette, while ACh was ejected from the micropipette with a 10-ms (positive) current pulse whose amplitude was adjusted to obtain ACh-gated depolarizations in the $\sim 10 \mathrm{mV}$ range. The ACh-filled micropipette was placed near an NMJ under visual control assisted by the use of f-PNA (see Section 5.9 below).

\subsection{Voltage-Clamp of X. laevis Oocytes Exogenously Expressing Muscle nAChRs from Zebrafish, Mouse, and Human}

cDNA clones of $\alpha-, \beta-(\beta 1 b$ isoform $), \gamma_{-}^{-}, \varepsilon-$, and $\delta$-subunits of the muscle subtypes of $n A C h R$ from zebrafish [25] were generously provided by Paul Brehm. cRNA were produced from these clones by linearizing with BamHI and transcription with T7 (mMessage mMachine T7 kit, Invitrogen, Carlsberg, 
CA, USA). cRNA injections and voltage-clamping of ACh-gated currents were done essentially as previously described [9]. Briefly, a given oocyte was injected with $\sim 1$ ng of cRNA for each nAChR subunit in the following combinations: $\alpha \beta \delta \gamma$ (fast embryonic muscle) or $\alpha \beta \delta \varepsilon$ (fast larvae and adult muscle) or $\alpha \beta \delta$ (slow muscle) and incubated at $16^{\circ} \mathrm{C}$ for at least one day. The same was done for the $\alpha \beta \varepsilon$ subunit combination except $\sim 10$ ng of cRNA were injected for each subunit. Preparation and injection into oocytes of the following materials were as previously described: cDNA for mouse and human muscle nAChR [55]; cRNA for rat $\alpha 3 \beta 4$ and $\alpha 4 \beta 2$ and human $\alpha 9 \alpha 10$ nAChRs $[9,86]$.

To record ACh-gated currents $\left(\mathrm{I}_{\mathrm{ACh}}\right)$, an oocyte was placed in a $30 \mu \mathrm{L}$ chamber, perfused with $\mathrm{ND} 96(96.0 \mathrm{mM} \mathrm{NaCl}, 2.0 \mathrm{mM} \mathrm{KCl}, 1.8 \mathrm{mM} \mathrm{CaCl} 2,1.0 \mathrm{mM} \mathrm{MgCl} 2,5 \mathrm{mM}$ Hepes, pH 7.1-7.5) containing $0.1 \mathrm{mg} / \mathrm{mL}$ bovine serum albumin, two-electrode voltage clamped at a holding potential of $-70 \mathrm{mV}$ with an OC725C Oocyte Clamp amplifier (Warner Instruments, Hamden, CT, USA), and exposed to a 1-s pulse of $\mathrm{ACh}(10 \mu \mathrm{M}$ for all nAChRs except zebrafish $\alpha \beta \varepsilon$-subunit combination where [ACh] was $100 \mu \mathrm{M}$ ) delivered at a frequency of $1 / \mathrm{min}$. Oocytes were exposed to toxin in a static bath for 5 -min. by halting the perfusion (and ACh pulses) and applying $3 \mu \mathrm{L}$ of toxin to the chamber at 10-times the final concentration and stirring the bath by aspirating and expelling the mixture with a pippetor. The same was done for control responses except $3 \mu \mathrm{L}$ ND96 was introduced into the static bath instead of peptide. After the five minutes had expired, the ACh pulses (and perfusion) were resumed. The peak amplitude of the first $\mathrm{I}_{\mathrm{ACh}}$ response after the 5-min ND96 control exposure served as the control response, and the peak amplitude of the first $\mathrm{I}_{\mathrm{ACh}}$ response after the 5-min toxin exposure served as the test response. The ratio of the test/control response was multiplied by 100 to obtain the percentage response. Each data point of a concentration-response curve represents the average \pm S.E. of at least three oocytes. Concentration-response curves were fit, with Prism software (GraphPad Software Inc., San Diego, $\mathrm{CA})$, to the Langmuir adsorption isotherm: \% Block $=100 \% /\left\{1+\left(\mathrm{IC}_{50} /[\right.\right.$ toxin] $\left.)\right\}$.

\subsection{Fluorescence Imaging}

5.9.1. Imaging of Fluorescently-Tagged $\alpha$-bungarotoxin ( $\alpha$-BgTX) and Fluorescein Peanut Agglutinin (f-PNA) Bound at NMJs

Tetramethylrhodamine (TMR) $\alpha$-BgTX and fluorescein PNA were used essentially as first described by the laboratories of Cohen and Ko, respectively [34,87]. TMR $\alpha$-BgTX and Alexa-488 $\alpha$-BgTX were purchased from Molecular Probes (Eugene, OR, USA) and used at concentrations and exposure times detailed in Results. Fluorescein-PNA was purchased from Vector Laboratories (Burlingame, CA, USA), and muscles were exposed to it at a concentration of $50 \mu \mathrm{g} / \mathrm{mL}$ for 20-30 min. f-PNA was originally used with R. pipiens NMJs, where it was shown not to adversely affect synaptic transmission [87]. We found that f-PNA also could serve in that capacity with X. laevis LP muscles (see Figure 13B); in contrast, we found that f-PNA was not useful as a marker for NMJs in fish.

Frog and goldfish muscle preparations were imaged with a Nikon FN-1 (upright) microscope fitted with a Nikon Apo 40X/0.8 water-immersion objective and a Nikon Digital Sight DS-Qi1Mc camera using a 2-s exposure at a gain of 8 . Zebrafish muscle preparations were imaged with a Nikon Ti (inverted) microscope fitted with Nikon Plan Fluor 40X/0.75 objective and a Retiga SRV-1394 camera (QImaging, Surrey, BC, Canada) with a 1-s exposure at a gain of 10. All images were acquired and processed with Nikon NIS-Elements software.

\subsubsection{Fluorescence Intensity Measurements}

The level of fluorescently tagged $\alpha$-BgTX bound at NMJs was quantified as follows. For frog muscles, a rectangular region of interest (ROI) with a width of 16 pixels $(2.56 \mu \mathrm{m})$ was placed over the NMJ with the ROI's long axis perpendicular to the long axis of the NMJ. A profile plot was obtained from the ROI where the plot's Y-axis represented fluorescence intensity averaged over the pixels in the short dimension of the ROI, and the plot's X-axis represented distance (in $\mu \mathrm{m}$ ) along the long dimension of the ROI (for an example, see Panels $\mathrm{C}$ and $\mathrm{H}$ in Figure 13). The value of the profile's peak 
minus that of the flanking baseline was taken as the fluorescence-intensity level. The same was done for fish muscles except the width of the rectangular ROI was narrower (6 pixels), and only NMJs were measured whose larger dimension exceeded the width of the ROI (see Figure S2B).

\subsection{Toxins and Their Application}

$\alpha$ M-MIIIJ was synthesized as described in Results. $\alpha$-conotoxin EI ( $\alpha$-EI), from C. ermineus, was synthesized as previously reported [22]. Toxins were dissolved in distilled water, NFR, NMR, or ND96, and stock solutions were kept frozen until use. Unless indicated otherwise, the solution bathing the preparation was manually removed and replaced by a toxin solution; static baths were used throughout to conserve toxin. Toxins were washed out by manually replacing the bath solution with NFR or NMR; in the case of oocytes, the bath was gravity perfused with ND96.

All experiments were performed at room temperature.

Supplementary Materials: The following are available online at http://www.mdpi.com/2072-6651/12/3/197/s1, Figure S1: HPLC elution profile of $C$. magus venom. Figure S2: $\alpha$ M-MIIIJ $(10 \mu \mathrm{M})$ inhibits fluorescently-tagged $\alpha$-BgTX binding at neuromuscular junctions of goldfish intercostal muscles, Figure S3: $\alpha \mathrm{M}-\mathrm{MIIIJ}(10 \mu \mathrm{M})$ inhibits binding of fluorescently-tagged $\alpha$-BgTX at both septal and distributed neuromuscular junctions in zebrafish, Table S1: Paralysis of goldfish induced by intramuscular injection of $\alpha \mathrm{M}-\mathrm{MIIIJ}$.

Author Contributions: Conceptualization, D.Y., J.M.M. and B.M.O.; methodology, M.J.R., H.O., I.B.L.R., L.A., J.M.M., H.S.-H., and D.Y.; validation, D.Y., J.M.M., and M.J.R., formal analysis D.Y., J.M.M., H.S.-H., and M.J.R.; investigation, D.Y., J.M.M., H.S.-H., and M.J.R.; writing-original draft preparation, D.Y., H.S.-H., and M.J.R.; writing-review and editing, M.J.R., H.O., I.B.L.R., L.A., J.M.M., H.S.H., B.M.O., and D.Y.; supervision, D.Y.; funding acquisition, J.M.M., D.Y., and B.M.O. All authors have read and agreed to the published version of the manuscript.

Funding: This research was funded by National Institute of General Medical Science grant (GM048677 to J.M.M., B.M.O., and D.Y. and GM122869 to B.M.O.). H.S.-H. acknowledges fellowship funding from the Velux Foundation (19063).

Acknowledgments: We thank our esteemed colleague and friend, Jean E. Rivier (1941-2019), for the chemical synthesis of $\alpha \mathrm{M}-\mathrm{MIIIJ}$ and many other conotoxins he synthesized over the past 4 decades. We also thank Paul Brehm for zebrafish nAChR clones, Ayako Yamaguchi for juvenile X. laevis frogs, Gabriel Bosse for zebrafish larvae and Kevin Chase for help with analysis of miniature EPSP amplitudes.

Conflicts of Interest: The authors declare no conflict of interest.

\section{References}

1. Olivera, B.M.; Raghuraman, S.; Schmidt, E.W.; Safavi-Hemami, H. Linking neuroethology to the chemical biology of natural products: Interactions between cone snails and their fish prey, a case study. J. Comp. Physiol. A 2017, 203, 717-735. [CrossRef] [PubMed]

2. Robinson, S.D.; Norton, R.S. Conotoxin gene superfamilies. Mar. Drugs 2014, 12, 6058-6101. [CrossRef] [PubMed]

3. Terlau, H.; Olivera, B.M. Conus Venoms: A Rich Source of Novel Ion Channel-Targeted Peptides. Physiol. Rev. 2004, 84, 41-68. [CrossRef] [PubMed]

4. King, G.F. Venoms as a platform for human drugs: Translating toxins into therapeutics. Expert Opin. Boil. Ther. 2011, 11, 1469-1484. [CrossRef] [PubMed]

5. Safavi-Hemami, H.; Brogan, S.E.; Olivera, B.M. Pain therapeutics from cone snail venoms: From Ziconotide to novel non-opioid pathways. J. Proteom. 2019, 190, 12-20. [CrossRef] [PubMed]

6. Olivera, B.M.; Seger, J.; Horvath, M.P.; Fedosov, A.E. Prey-Capture Strategies of Fish-Hunting Cone Snails: Behavior, Neurobiology and Evolution. Brain Behav. Evol. 2015, 86, 58-74. [CrossRef] [PubMed]

7. Olivera, B.M. Conus Venom Peptides, Receptor and Ion Channel Targets, and Drug Design: 50 Million Years of Neuropharmacology. Mol. Biol. Cell 1997, 8, 2101-2109. [CrossRef]

8. McIntosh, M.; Cruz, L.J.; Hunkapiller, M.W.; Gray, W.R.; Olivera, B.M. Isolation and structure of a peptide toxin from the marine snail Conus magus. Arch. Bioch. Biophys. 1982, 218, 329-334. [CrossRef]

9. Cartier, G.E.; Yoshikami, D.; Gray, W.R.; Luo, S.; Olivera, B.M.; McIntosh, J.M. A new alpha-conotoxin which targets alpha3beta2 nicotinic acetylcholine receptors. J. Biol. Chem. 1996, 271, 7522-7528. [CrossRef] 
10. Olivera, B.M.; Cruz, L.J.; de Santos, V.; LeCheminant, G.W.; Griffin, D.; Zeikus, R.; McIntosh, J.M.; Galyean, R.; Varga, J.; Gray, W.R. Neuronal calcium channel antagonists. Discrimination between calcium channel subtypes using omega-conotoxin from Conus magus venom. Biochemistry 1987, 26, 2086-2090. [CrossRef]

11. Wilson, M.J.; Yoshikami, D.; Azam, L.; Gajewiak, J.; Olivera, B.M.; Bulaj, G.; Zhang, M.M. mu-Conotoxins that differentially block sodium channels NaV1.1 through 1.8 identify those responsible for action potentials in sciatic nerve. Proc. Natl. Acad. Sci. USA 2011, 108, 10302-10307. [CrossRef] [PubMed]

12. Zhang, M.-M.; Fiedler, B.; Green, B.R.; Catlin, P.; Watkins, M.; Garrett, J.E.; Smith, B.J.; Yoshikami, J.; Olivera, B.M.; Bulaj, G.; et al. Structural and Functional Diversities among $\mu$-Conotoxins Targeting TTX-resistant Sodium Channels. Biochemistry 2006, 45, 3723-3732. [CrossRef] [PubMed]

13. Sato, S.; Nakamura, H.; Ohizumi, Y.; Kobayashi, J.; Hirata, Y. The amino acid sequences of homologous hydroxyproline-containing myotoxins from the marine snail Conus geographus venom. FEBS Lett. 1983, 155, 277-280. [CrossRef]

14. Violette, A.; Biass, D.; Dutertre, S.; Koua, D.; Piquemal, D.; Pierrat, F.; Stöcklin, R.; Favreau, P. Large-scale discovery of conopeptides and conoproteins in the injectable venom of a fish-hunting cone snail using a combined proteomic and transcriptomic approach. J. Proteom. 2012, 75, 5215-5225. [CrossRef]

15. Bulaj, G.; West, P.J.; Garrett, J.E.; Watkins, M.; Zhang, M.-M.; Norton, R.S.; Smith, B.J.; Yoshikami, J.; Olivera, B.M. Novel Conotoxins from Conus striatusandConus kinoshitaiSelectively Block TTX-Resistant Sodium Channels. Biochemistry 2005, 44, 7259-7265. [CrossRef]

16. Holford, M.; Zhang, M.-M.; Gowd, K.H.; Azam, L.; Green, B.R.; Watkins, M.; Ownby, J.-P.; Yoshikami, J.; Bulaj, G.; Olivera, B.M.; et al. Pruning nature: Biodiversity-derived discovery of novel sodium channel blocking conotoxins from Conus bullatus. Toxicon 2008, 53, 90-98. [CrossRef]

17. Li, Q.; Watkins, M.; Robinson, S.D.; Safavi-Hemami, H.; Yandell, M. Discovery of Novel Conotoxin Candidates Using Machine Learning. Toxins 2018, 10, 503. [CrossRef]

18. Lluisma, A.O.; Vera, E.L.; Bulaj, G.; Watkins, M.; Olivera, B.M. Characterization of a novel $\psi$-conotoxin from Conus parius Reeve. Toxicon 2007, 51, 174-180. [CrossRef]

19. Safo, P.; Rosenbaum, T.; Shcherbatko, A.; Choi, D.Y.; Han, E.; Toledo-Aral, J.J.; Olivera, B.M.; Brehm, P.; Mandel, G. Distinction among neuronal subtypes of voltage-activated sodium channels by mu-conotoxin PIIIA. J. Neurosci. 2000, 20, 76-80. [CrossRef]

20. Shon, K.J.; Grilley, M.; Jacobsen, R.; Cartier, G.E.; Hopkins, C.; Gray, W.R.; Watkins, M.; Hillyard, D.R.; Rivier, J.; Torres, J.; et al. A noncompetitive peptide inhibitor of the nicotinic acetylcholine receptor from Conus purpurascens venom. Biochemistry 1997, 36, 9581-9587. [CrossRef]

21. Jacob, R.B.; McDougal, O.M. The M-superfamily of conotoxins: A review. Cell. Mol. Life Sci. 2009, 67, 17-27. [CrossRef] [PubMed]

22. Martinez, J.S.; Olivera, B.M.; Gray, W.R.; Craig, A.G.; Groebe, D.R.; Abramson, S.N.; McIntosh, J.M. alpha-Conotoxin EI, a new nicotinic acetylcholine receptor antagonist with novel selectivity. Biochemistry 1995, 34, 14519-14526. [CrossRef] [PubMed]

23. Shon, K.-J.; Olivera, B.M.; Watkins, M.; Jacobsen, R.B.; Gray, W.R.; Floresca, C.Z.; Cruz, L.J.; Hillyard, D.R.; Brink, A.; Terlau, H.; et al. $\mu$-Conotoxin PIIIA, a New Peptide for Discriminating among Tetrodotoxin-Sensitive Na Channel Subtypes. J. Neurosci. 1998, 18, 4473-4481. [CrossRef] [PubMed]

24. McLachlan, E.M.; Martin, A.R. Non-linear summation of end-plate potentials in the frog and mouse. J. Physiol. 1981, 311, 307-324. [CrossRef]

25. Mongeon, R.; Walogorsky, M.; Urban, J.; Mandel, G.; Ono, F.; Brehm, P. An acetylcholine receptor lacking both $\gamma$ and $\varepsilon$ subunits mediates transmission in zebrafish slow muscle synapses. J. Gen. Physiol. 2011, 138, 353-366. [CrossRef]

26. Drapeau, P.; Buss, R.R.; Ali, D.W.; Legendre, P.; Rotundo, R.L. Limits to the development of fast neuromuscular transmission in zebrafish. J. Neurophysiol. 2001, 86, 2951-2956. [CrossRef]

27. Nguyen, P.V.; Aniksztejn, L.; Catarsi, S.; Drapeau, P. Maturation of neuromuscular transmission during early development in zebrafish. J. Neurophysiol. 1999, 81, 2852-2861. [CrossRef]

28. López-Vera, E.; Aguilar, M.B.; Schiavon, E.; Marinzi, C.; Ortiz, E.; Restano Cassulini, R.; Batista, C.V.; Possani, L.D.; Heimer de la Cotera, E.P.; Peri, F.; et al. Novel alpha-conotoxins from Conus spurius and the alpha-conotoxin EI share high-affinity potentiation and low-affinity inhibition of nicotinic acetylcholine receptors. FEBS J. 2007, 274, 3972-3985. [CrossRef] 
29. Jimenez, E.C.; Olivera, B.M.; Teichert, R.W. AlphaC-conotoxin PrXA: A new family of nicotinic acetylcholine receptor antagonists. Biochemistry 2007, 46, 8717-8724. [CrossRef]

30. Luna, V.M.; Daikoku, E.; Ono, F. “Slow” skeletal muscles across vertebrate species. Cell Biosci. 2015, 5, 62. [CrossRef]

31. Green, B.R.; Olivera, B.M. Venom Peptides From Cone Snails: Pharmacological Probes for Voltage-Gated Sodium Channels. Curr. Top. Membr. 2016, 78, 65-86. [CrossRef] [PubMed]

32. Kerr, L.M.; Yoshikami, D.A. Venom peptide with a novel presynaptic blocking action. Nature 1984, 308, 282-284. [CrossRef] [PubMed]

33. Jacobsen, R.; Yoshikami, D.; Ellison, M.; Martinez, J.; Gray, W.R.; Cartier, G.E.; Shon, K.J.; Groebe, D.R.; Abramson, S.N.; Olivera, B.M.; et al. Differential targeting of nicotinic acetylcholine receptors by novel alphaA-conotoxins. J. Biol. Chem. 1997, 272, 22531-22537. [CrossRef] [PubMed]

34. Anderson, M.J.; Cohen, M.W. Fluorescent staining of acetylcholine receptors in vertebrate skeletal muscle. J. Physiol. 1974, 237, 385-400. [CrossRef]

35. Tarvin, R.D.; Borghese, C.M.; Sachs, W.; Santos, J.C.; Lu, Y.; Connell, L.A.; Cannatella, D.C.; Harris, R.A.; Zakon, H.H. Interacting amino acid replacements allow poison frogs to evolve epibatidine resistance. Science 2017, 357, 1261-1266. [CrossRef] [PubMed]

36. Tavazoie, S.F.; Tavazoie, M.F.; McIntosh, J.M.; Olivera, B.M.; Yoshikami, D. Differential block of nicotinic synapses on B versus C neurones in sympathetic ganglia of frog by alpha-conotoxins MII and ImI. Br. J. Pharmacol. 1997, 120, 995-1000. [CrossRef] [PubMed]

37. Gray, W.R.; Luque, A.; Olivera, B.M. Peptide Toxins from Conus geographus Venom. J. Biol. Chem. 1981, 256, 4734-4740.

38. Groebe, D.R.; Dumm, J.M.; Levitan, E.S.; Abramson, S.N. alpha-Conotoxins selectively inhibit one of the two acetylcholine binding sites of nicotinic receptors. Mol. Pharmacol. 1995, 48, 105-111.

39. Kapono, C.A.; Thapa, P.; Cabalteja, C.C.; Guendisch, D.; Collier, A.C.; Bingham, J.-P. Conotoxin truncation as a post-translational modification to increase the pharmacological diversity within the milked venom of Conus magus. Tox. Off. J. Int. Soc. Toxinol. 2013, 70, 170-178. [CrossRef]

40. McIntosh, J.M.; Dowell, C.; Watkins, M.; Garrett, J.E.; Yoshikami, D.; Olivera, B.M. a-Conotoxin GIC from Conus geographus, a Novel Peptide Antagonist of Nicotinic Acetylcholine Receptors. J. Biol. Chem. 2002, 277, 33610-33615. [CrossRef]

41. Zafaralla, G.C.; Ramilo, C.; Gray, W.R.; Karlstrom, R.; Olivera, B.M.; Cruz, L.J. Phylogenetic specificity of cholinergic ligands: Alpha-conotoxin SI. Biochemistry 1988, 27, 7102-7105. [CrossRef] [PubMed]

42. Myers, R.A.; Zafaralla, G.C.; Gray, W.R.; Abbott, J.; Cruz, L.J.; Olivera, B.M. alpha-Conotoxins, small peptide probes of nicotinic acetylcholine receptors. Biochemistry 1991, 30, 9370-9377. [CrossRef] [PubMed]

43. Ramilo, C.A.; Zafaralla, G.C.; Nadasdi, L.; Hammerland, L.G.; Yoshikami, D.; Gray, W.R.; Kristipati, R.; Ramachandran, J.; Miljanich, G.; Olivera, B.M. Novel alpha- and omega-conotoxins from Conus striatus venom. Biochemistry 1992, 31, 9919-9926. [CrossRef] [PubMed]

44. Liu, L.; Chew, G.; Hawrot, E.; Chi, C.; Wang, C. Two potent alpha3/5 conotoxins from piscivorous Conus achatinus. Acta Biochim. Biophys. Sinica 2007, 39, 438-444. [CrossRef]

45. Favreau, P.; Krimm, I.; Le Gall, F.; Bobenrieth, M.J.; Lamthanh, H.; Bouet, F.; Servent, D.; Molgo, J.; Ménez, A.; Letourneux, Y.; et al. Biochemical characterization and nuclear magnetic resonance structure of novel alpha-conotoxins isolated from the venom of Conus consors. Biochemistry 1999, 38, 6317-6326. [CrossRef]

46. Nicke, A.; Loughnan, M.L.; Millard, E.L.; Alewood, P.F.; Adams, D.J.; Daly, N.L.; Craik, D.J.; Lewis, R.J. Isolation, structure, and activity of GID, a novel alpha 4/7-conotoxin with an extended N-terminal sequence. J. Biol. Chem. 2003, 278, 3137-3144. [CrossRef]

47. Dowell, C.; Olivera, B.M.; Garrett, J.E.; Staheli, S.T.; Watkins, M.; Kuryatov, A.; Yoshikami, D.; Lindstrom, J.M.; McIntosh, J.M. a-Conotoxin PIA Is Selective for a6 Subunit-Containing Nicotinic Acetylcholine Receptors. J. Neurosci. 2003, 23, 8445-8452. [CrossRef]

48. McIntosh, J.M.; Plazas, P.V.; Watkins, M.; Gomez-Casati, M.E.; Olivera, B.M.; Elgoyhen, A.B. A Novel $\alpha$-Conotoxin, PeIA, Cloned from Conus pegrandis, Discriminates between Rat $\alpha 9 \alpha 10$ and $\alpha 7$ Nicotinic Cholinergic Receptors. J. Biol. Chem. 2005, 34, 30107-30112. [CrossRef]

49. Azam, L.; Dowell, C.; Watkins, M.; Stitzel, J.A.; Olivera, B.M.; McIntosh, J.M. $\alpha$-Conotoxin BuIA, a Novel Peptide from Conus bullatus, distinguishes among Neuronal Nicotinic Acetylcholine Receptors. J. Biol. Chem. 2005, 280, 80-87. [CrossRef] 
50. Lopez-Vera, E.; Jacobsen, R.B.; Ellison, M.; Olivera, B.M.; Teichert, R.W. A novel alpha conotoxin (a-PIB) isolated from C. purpurascens is selective for skeletal muscle nicotinic acetylcholine receptors. Toxicon 2007, 49, 1193-1199. [CrossRef]

51. Quinton, L.; Servent, D.; Girard, E.; Molgó, J.; Caer, J.-P.L.; Malosse, C.; Haidar, E.A.; Lecoq, A.; Gilles, N.; Chamot-Rooke, J.; et al. Identification and functional characterization of a novel $\alpha$-conotoxin (EIIA) from Conus ermineus. Anal. Bioanal. Chem. 2013, 405, 5341-5351. [CrossRef] [PubMed]

52. Hopkins, C.; Grilley, M.; Miller, C.; Shon, K.J.; Cruz, L.J.; Gray, W.R.; Dykert, J.; Rivier, J.; Yoshikami, D.; Olivera, B.M.; et al. A New Family of Conus Peptides Targeted to the Nicotinic Acetylcholine-Receptor. J. Biol. Chem. 1995, 270, 22361-22367. [CrossRef] [PubMed]

53. Teichert, R.W.; Rivier, J.; Dykert, J.; Cervini, L.; Gulyas, J.; Bulaj, G.; Ellison, M.; Olivera, B.M. AlphaA-Conotoxin OIVA defines a new alphaA-conotoxin subfamily of nicotinic acetylcholine receptor inhibitors. Toxicon 2004, 44, 207-214. [CrossRef] [PubMed]

54. Teichert, R.W.; Jimenez, E.C.; Olivera, B.M. Alpha S-contoxin RVIIIA: A structurally unique conotoxin that broadly targets nicotinic acetylcholine receptors. Biochemistry 2005, 44, 7897-7902. [CrossRef] [PubMed]

55. Teichert, R.W.; Lopez-Vera, E.; Gulyas, J.; Watkins, M.; Rivier, J.; Olivera, B.M. Definition and characterization of the short alphaA-conotoxins: A single residue determines dissociation kinetics from the fetal muscle nicotinic acetylcholine receptor. Biochemistry 2006, 45, 1304-1312. [CrossRef]

56. Christensen, S.B.; Bandyopadhyay, P.K.; Olivera, B.M.; McIntosh, J.M. alphaS-conotoxin GVIIIB potently and selectively blocks alpha9alpha10 nicotinic acetylcholine receptors. Biochem. Pharmacol. 2015, 96, 349-356. [CrossRef]

57. Van Wagoner, R.M.; Jacobsen, R.B.; Olivera, B.M.; Ireland, C.M. Characterization and three-dimensional structure determination of psi-conotoxin Piiif, a novel noncompetitive antagonist of nicotinic acetylcholine receptors. Biochemistry 2003, 42, 6353-6362. [CrossRef]

58. Ellison, M.; McIntosh, J.M.; Olivera, B.M. Alpha-conotoxins ImI and ImII. Similar alpha 7 nicotinic receptor antagonists act at different sites. J. Biol. Chem. 2003, 278, 757-764. [CrossRef]

59. Johnson, D.S.; Martinez, J.; Elgoyhen, A.B.; Heinemann, S.F.; McIntosh, J.M. Alpha-Conotoxin ImI exhibits subtype-specific nicotinic acetylcholine receptor blockade: Preferential inhibition of homomeric alpha 7 and alpha 9 receptors. Mol. Pharmacol. 1995, 48, 194-199.

60. Ellison, M.; Gao, F.; Wang, H.L.; Sine, S.M.; McIntosh, J.M.; Olivera, B.M. Alpha-conotoxins ImI and ImII target distinct regions of the human alpha7 nicotinic acetylcholine receptor and distinguish human nicotinic receptor subtypes. Biochemistry 2004, 43, 16019-16026. [CrossRef]

61. Ellison, M.; Olivera, B.M. Alpha4/3 conotoxins: Phylogenetic distribution, functional properties, and structure-function insights. Chem. Rec. 2007, 7, 341-353. [CrossRef]

62. McIntosh, J.M.; Hasson, A.; Spira, M.E.; Gray, W.R.; Li, W.; Marsh, M.; Hillyard, D.R.; Olivera, B.M. A new family of conotoxins that blocks voltage-gated sodium channels. J. Biol. Chem. 1995, 270, 16796-16802. [CrossRef] [PubMed]

63. Fainzilber, M.; van der Schors, R.; Lodder, J.C.; Li, K.W.; Geraerts, W.P.; Kits, K.S. New sodium channel-blocking conotoxins also affect calcium currents in Lymnaea neurons. Biochemistry 1995, 34, 5364-5371. [CrossRef] [PubMed]

64. Bulaj, G.; Zhang, M.-M.; Green, B.R.; Fiedler, B.; Layer, R.T.; Wei, S.; Nielsen, J.S.; Low, S.J.; Klein, B.D.; Wagstaff, J.D.; et al. Synthetic muO-conotoxin MrVIB blocks TTX-resistant sodium channel NaV1.8 and has a long-lasting analgesic activity. Biochemistry 2006, 45, 7404-7414. [CrossRef] [PubMed]

65. Ekberg, J.; Jayamanne, A.; Vaughan, C.W.; Aslan, S.; Thomas, L.; Mould, J.; Drinkwater, R.; Baker, M.D.; Abrahamsen, B.; Wood, J.N.; et al. muO-conotoxin MrVIB selectively blocks Nav1.8 sensory neuron specific sodium channels and chronic pain behavior without motor deficits. Proc. Natl Acad. Sci. USA 2006, 103, 17030-17035. [CrossRef] [PubMed]

66. Terlau, H.; Stocker, M.; Shon, K.J.; McIntosh, J.M.; Olivera, B.M. mu O-conotoxin MrVIA inhibits mammalian sodium channels, but not through site I. J. Neurophys. 1996, 76, 1423-1429. [CrossRef]

67. Favreau, P.; Benoit, E.; Hocking, H.G.; Carlier, L.; D’hoedt, D.; Leipold, E.; Markgraf, R.; Schlumberger, S.; Córdova, M.A.; Gaertner, H.; et al. A novel $\mu$-conopeptide, CnIIIC, exerts potent and preferential inhibition of NaV1.2/1.4 channels and blocks neuronal nicotinic acetylcholine receptors. Br. J. Pharmacol. 2012, 166, 1654-1668. [CrossRef] 
68. Leipold, E.; Ullrich, F.; Thiele, M.; Tietze, A.A.; Terlau, H.; Imhof, D.; Heinemann, S.H. Subtype-specific block of voltage-gated $\mathrm{K}(+)$ channels by $\mu$-conopeptides. Biochem. Biophys. Res. Commun. 2016. [CrossRef]

69. Liu, Z.; Bartels, P.; Sadeghi, M.; Du, T.; Dai, Q.; Zhu, C.; Yu, S.; Wang, S.; Dong, M.; Sun, T.; et al. A novel $\alpha$-conopeptide Eu1.6 inhibits N-type (CaV2.2) calcium channels and exhibits potent analgesic activity. Sci. Rep. 2018, 8, 1004. [CrossRef]

70. Andreson, R.; Roosaare, M.; Kaplinski, L.; Laht, S.; Kõressaar, T.; Lepamets, M.; Brauer, A.; Kukuškina, V.; Remm, M. Gene content of the fish-hunting cone snail Conus consors. bioRxiv 2020. [CrossRef]

71. Altschul, S.F.; Gish, W.; Miller, W.; Myers, E.W.; Lipman, D.J. Basic local alignment search tool. J. Mol. Biol. 1990, 215, 403-410. [CrossRef]

72. Stewart, J.; Young, J. Solid Phase Peptide Synthesis; Pierce Chemical Company: Rockford, IL, USA, 1984.

73. Horiki, K.; Igano, K.; Inouye, K. Amino acids and peptides. Part 6. Synthesis of the Merrifield resin esters of $\mathrm{N}$-protected amino acids with the aid of hydrogen bonding. Chem. Lett. 1978, 2, 165-168. [CrossRef]

74. Kaiser, E.; Colescott, R.; Bossinger, C.; Cook, P. Color test for detection of free terminal amino groups in the solid-phase synthesis of peptides. Anal. Biochem. 1970, 34, 595-598. [CrossRef]

75. Ellman, G. Tissue sulfhydryl groups. Arch. Biochem. Biophys. 1959, 82, 70-77. [CrossRef]

76. Betz, W.J.; Bewick, G.S. Optical analysis of synaptic vesicle recycling at the frog neuromuscular junction. Science 1992, 255, 200-203. [CrossRef] [PubMed]

77. Peper, K.; McMahan, U.J. Distribution of acetylcholine receptors in the vincinity of nerve terminals on skeletal muscle of the frog. Proc. R. Soc. B. 1972, 181, 431-440.

78. Jimenez, E.C.; Shetty, R.P.; Lirazan, M.; Rivier, J.; Walker, C.; Abogadie, F.C.; Yoshikami, D.; Cruz, L.J.; Olivera, B.M. Novel excitatory Conus peptides define a new conotoxin superfamily. J. Neurochem. 2003, 85, 610-621. [CrossRef]

79. Gilchrist, J.D.F.; von Bonde, C. Dissection of the Platana and the Frog; Good Press: Glasgow, UK, 1919 ; p. 8. [CrossRef]

80. Brady, J. A simple technique for making very fine, durable dissecting needles by sharpening tungsten wire electrolytically. Bull. WHO 1965, 32, 143-144.

81. Li, W.; Ono, F.; Brehm, P. Optical measurements of presynaptic release in mutant zebrafish lacking postsynaptic receptors. J. Neurosci. 2003, 23, 10467-10474. [CrossRef]

82. Ono, F.; Higashijima, S.; Shcherbatko, A.; Fetcho, J.R.; Brehm, P. Paralytic zebrafish lacking acetylcholine receptors fail to localize rapsyn clusters to the synapse. J. Neurosci. 2001, 21, 5439-5448. [CrossRef]

83. Angaut-Petit, D.; Molgó, J.; Connold, A.L.; Faille, L. The levator auris longus muscle of the mouse: A convenient preparation for studies of short-and long-term presynaptic effects of drugs or toxins. Neurosci. Lett. 1987 82, 83-88. [CrossRef]

84. Kuffler, S.W.; Yoshikami, D. The distribution of acetylcholine sensitivity at the post-synaptic membrane of vertebrate skeletal twitch muscles: Iontophoretic mapping in the micron range. J. Physiol. 1975, 244, 703-730. [CrossRef] [PubMed]

85. Kamata, R.; Washio, H. Neuromuscular transmission of pectoral fin muscles of the goldfish Carassius auratus. Comp. Biochem. Physiol. Part A Mol. Integr. Physiol. 2003, 135, 435-442. [CrossRef]

86. Filchakova, O.; McIntosh, J.M. Functional expression of human $\alpha 9^{*}$ nicotinic acetylcholine receptors in X. laevis oocytes is dependent on the $\alpha 9$ subunit 5' UTR. PLoS ONE 2013, 8, e64655. [CrossRef] [PubMed]

87. Ko, C.P. A lectin, peanut agglutinin, as a probe for the extracellular matrix in living neuromuscular junctions. J. Neurocytol. 1987, 16, 567-576. [CrossRef] [PubMed]

(C) 2020 by the authors. Licensee MDPI, Basel, Switzerland. This article is an open access article distributed under the terms and conditions of the Creative Commons Attribution (CC BY) license (http://creativecommons.org/licenses/by/4.0/). 